\title{
GLOBAL IDEAL THEORY OF MEROMORPHIC FUNCTION FIELDS \\ BY
}

NORMAN L. ALLING

\begin{abstract}
It is shown that the ideal theories of the fields of all meromorphic functions on any two noncompact Riemann surfaces are isomorphic. Further, various new representation and factorization theorems are proved.
\end{abstract}

Introduction. Throughout this paper let $X$ and $Y$ denote noncompact (connected) Riemann surfaces. Let $A(X)$ (or $A$ for short), denote the ring of all analytic functions on $X$, and let $F(X)$ (or $F$ for short), denote the field of all meromorphic functions on $X$. In 1940 Helmer [10] studied divisibility properties in $A(C)$, laid the foundations for its ideal theory, and proved that every finitely generated ideal in it is principal. (See [2, pp. 24-28] for a brief history of the subject from 1940 to 1966.) In 1952-53 Henriksen [11], [12] investigated the maximal and prime ideals of $A(C)$, finding-among other things-that each prime ideal is contained in a unique maximal ideal. An ideal of a ring will be called local if it is contained in a unique maximal ideal; thus Henriksen proved that each prime ideal of $A(C)$ is local. In 1948 Florack [7] proved essentially that $X$ is a Stein manifold. Using her theorem, the investigation of the ideal theory of $A(X)$, for $X \subset \mathrm{C}$, was gradually generalized to arbitrary $X$. In 1963 the author [1] showed that if $M$ is a maximal ideal of $A$ then the ring of quotients, $A_{M}$, is a valuation ring. At that time the value group of $A_{M}$ was also investigated. Using classical methods of commutative algebra, one can make a very complete analysis of the local ideals of $A$.

The initial aim of this research was to learn more about the decomposition of an ideal $I$ of $A$ as an intersection of local ideals. In trying to extend local knowledge to obtain global results it became evident that some topology on the set specm $A$ of maximal ideals was needed. The author turned, naturally, to the Zariski topology on specm $A . X$ is, in a natural way, identifiable with a subset of specm $A$. Let $X_{0}$ be the topology induced on $X$ by this identification; it will be called the zero set topology on $X$. It is obvious that $X_{0}$ is a much coarser topology than $X$. The author was surprised to learn (1.3) that $X_{0}$ and $Y_{0}$ are always homeomorphic. One possible inference to be drawn is

Received by the editors December 1, 1978.

AMS (MOS) subject classifications (1970). Primary 13A15, 30 A98.

Key words and phrases. Ideals, submodules, subrings, divisors, valuations, lattices, dual ideals, meromorphic functions, analytic functions, Riemann surfaces. 
that the zero set topology is not very interesting. One of the main purposes of this paper is to see how much information about $A$ and $F$ is carried by the zero set topology. One immediately finds (1.8) that specm $A(X)$ and specm $A(Y)$ are always homeomorphic. In $\$ 2$ the group Div $X$ of divisors of $F(X)$ is considered. (1.3) immediately implies that $\operatorname{Div} X$ and $\operatorname{Div} Y$ are always isomorphic as lattice-ordered groups (2.3). Let $\nu$ map $f \in F(X)$ to its divisor in $(\operatorname{Div} X) \cup\{\infty\}$. In 1946 Schilling [18] considered fractional ideals in $F(C)$. Generalizing his work we consider, in $\$ 2$, the set of all sub $A$-modules of $F, \operatorname{sam}(F)$; the set of all fractional ideals of $F, I(F)$; and the set of all ideals of $A, I(A)$. Given $I \in \operatorname{sam}(F)$ it turns out (2.6) that $\nu(I)$ is a dual ideal in the lattice (Div $X) \cup\{\infty\}$, i.e., an element in $\operatorname{di}(\operatorname{Div} X)$. In fact, $\nu$ is a bijection of $\operatorname{sam}(F)$ onto $\operatorname{di}(\operatorname{Div} X)$; that maps $I(F)$ onto $J(\operatorname{Div} X)$, elements of $\operatorname{di}(\operatorname{Div} X)$ that are bounded below; and $I(A)$ to elements $J\left(\mathrm{Div}^{+} X\right)$ of $\operatorname{di}(\operatorname{Div} X)$ that are bounded below by zero. Pursuing further some suggestions found in Banaschewski's paper [4] of 1958, it is also shown, in \$2, that all the classical ideal theory of $F$ is bijectively carried over by $\nu$ to di(Div $X)$. One of the main conclusions of this analysis is that the ideal theory of $A(X)$ is always isomorphic to that of $A(Y)$, and the ideal theory of $F(X)$ is always isomorphic to the ideal theory of $F(Y)$ (2.13). This seems, at first sight, to be in sharp contrast with the Chevalley-Kakutani, Bers Theorem [5] (which states that $A(X)$ and $A(Y)$ are C-isomorphic if and only if $X$ and $Y$ are conformally equivalent), and Iss'sa's Theorem [14] (which states that $F(X)$ and $F(Y)$ are C-isomorphic if and only if $X$ and $Y$ are conformally equivalent). In $\$ 3$ we discuss the local ideal theory of $A$, obtaining a few new results and setting the stage for \$4. One advantage of dealing with dual ideals in (Div $X) \cup\{\infty\}$ rather than sub $A$-modules of $F$ is that the dual ideals are easy to characterize, as we will see in \$4. In \$4 we first exploit the fact that for each $M \in \operatorname{specm} A, A_{M}$ is a valuation ring, to extend each $a \in \operatorname{Div} X$ to $\tilde{a}$ a map of $\delta X$ (a compactification of $X_{0}$ homeomorphic to specm $A$ ) into $\Pi_{\mu \in \delta X} G_{\mu}$, where $G_{\mu}$ is the value group of $A_{M}, M$ being naturally associated with $\mu$. Next we consider the Dedekind completion $H_{\mu}$ of $G_{\mu}$, and let $H \equiv \Pi_{\mu \in \delta x} H_{\mu}$; then $H$ is a complete lattice. $h \in H$ will be said to be approximable from above by divisors if $h=\bigwedge_{\tilde{a}>h} \tilde{a}$, where $a \in \operatorname{Div} X$. Let afa $(H)$ be the set of all such $h \in H$. Given $J \in \operatorname{di}(\operatorname{Div} X)$, let $L(J) \equiv \bigwedge_{j \in J} \equiv h$; then $h \in \operatorname{afa}(H)$ and $J=\{a \in(\operatorname{Div} X) \cup\{\infty\}: \tilde{a}>h\}$, (4.3). $h$ will be called the virtual generator of $J$. Further $L \circ \nu$ is a bijection of $\operatorname{sam}(F)$ onto afa $(H)$; then $I(F)$ maps onto $\operatorname{afa}^{*}(H)(\equiv\{h \in \mathrm{afa}(H)$ : there exists $a \in \operatorname{Div} X$ for which $h>\tilde{a}\})$; and $I(A)$ maps onto afa $\left(H^{+}\right)(\equiv\{h \in \operatorname{afa}(H): h>0\})$, (4.4). Finally $L \circ \nu$ preserve the operations of classical ideal theory (4.5). Applications and further developments occupy $\$ 5$. The main factorization theorem (5.6) states that any nonzero fractional ideal in $F$ is the product of a principal ideal in $F$ and a free ideal in $A$. Further this representation is unique. Schilling's 
Theorem [18], that states that every closed ideal of $A$ is principal, is generalized from $C$ to $X$ (5.14). The final topic to be discussed in $\$ 5$ is that of subrings $B$ of $F$ that contain $A$, which Kelleher [15] called $A$-rings. Using the virtual-generator $h$ of $B$, we define a convex subgroup $\Gamma$ of $\operatorname{Div} X$, which turns out to be $\nu(U(B)), U(B)$ being the group of units of $B$. With the aid of $\Gamma$ a complete analysis of the ideal theory of $B$ along the lines presented in $\S 2-\S 4$ can be made.

1. The zero set topology on $X$. In her 1948 Münster Dissertation [7], Herta Florack proved that a general Mittag-Leffler and a Weierstrass (product) theorem holds on $X$. This result gives us almost all we need, as to existence theorems on $X$. The imbedding theorem will also be used, thus it is well to quote the cardinal result: namely that $X$ is a Stein manifold. (See e.g. [9, p. 270] for details.) Then it follows that Cartan's Theorem B holds for $X$; from which Florack's Theorem follows. $A$ is-of course-an integral domain, every element of $F$ is a quotient of elements of $A$, and $A$ is integrally closed in $F$.

Given an integral domain $B$, let $I(B)$ denote the set of all ideals of $B$, ordered under inclusion. Let prop $I(B) \equiv I(B)-\{B\}$. Let spec $B$ denote the set of all proper prime ideals of $B$, under the Zariski topology; and let specm $B$ the subspace of all maximal ideals of $B$. Each of these spaces is compact. spec $B$ is $T_{0}$, but is usually not $T_{1}$. specm $B$ is $T_{1}$ but is usually not $T_{2}$. Given $I \in I(B)$, let $V(I)$, the variety of $I$, be $\{M \in \operatorname{specm} B: I \subset M\}$; then $\{V(I): I \in I(B)\}$ is-by definition-the set of closed sets of specm $B$, and $\{V((f)): f \in B\}$ is a basis for the closed sets of specm $B$.

For each $x \in X$, let $M_{x} \equiv\{f \in A: f(x)=0\} \equiv m(x)$. By Florack's Theorem, $m$ is an injection of $X$ into specm $A(X)$. Let $X_{0}$ denote the set $X$ with the topology which makes $m$ a homeomorphism of $X$ onto $m(X)$. For $f \in A$ let $Z(f) \equiv\{x \in X: f(x)=0\}$, and let this set be called the zero set of $f$. For any $S \subset A$ let $Z(S) \equiv\{Z(f): f \in S\}$. Let $\Delta(X)$ (or $\Delta$ for short) be $Z(A(X))$, and let prop $\Delta(X) \equiv \Delta(X)-\{X\}$. By Florack's Theorem, $\Delta(X)$ is the set of all closed discrete subsets of $X$. Clearly

$$
Z(f)=m^{-1}(V((f)) \cap m(X)) ;
$$

thus $\Delta(X)$ is a basis for the set of closed sets of $X_{0}$. Since $\Delta(X)$ is closed under intersection, $\Delta(X)$ is the set of all closed subsets of $X_{0}$. The topology $X_{0}$ will be called the zero set topology on $X . X_{0}$ is a noncompact $T_{1}$-space, which is not $T_{2}$. (The following was noted by the author's colleague, Professor A. H. Stone, during a very useful conversation about this research in the Fall of 1977.)

LEMMA 1.1. Let $K$ be a compact subset of $X$ and let $K_{0}$ denote this set with the topology induced from $X_{0}$. The proper closed subsets of $K_{0}$ are just the finite subsets of $K_{0}$; thus $K_{0}$ has the cofinite topology on it. 
Proof. Given $D \in$ prop $\Delta, D \cap K$ is compact and discrete in $X$, and hence it is finite.

LEMMA 1.2. There exists a family $\left(U_{n}\right)_{n \in N}$ of nonempty, open, relatively compact subsets of $X$ that cover $X$ such that $\bar{U}_{n} \varsubsetneqq U_{n+1}$, for each $n \in N$.

Proof. Since $X$ is a Stein manifold, we may apply the imbedding theorem (see e.g. [9, pp. 219-226]), and thus find an analytic homeomorphism $f$ of $X$ onto a closed submanifold of $C^{4}$. Translate $f(X)$ in $C^{4}$ so that $0 \in f(X)$. For each $n \in N$ let

$$
U_{n} \equiv f^{-1}\left(\left\{z \in \mathbf{C}^{4}:\|z\|<n\right\}\right) .
$$

Then $\left(U_{n}\right)_{n \in N}$ has the required properties.

THEOREM 1.3. Let $X$ and $Y$ be noncompact (connected) Riemann surfaces; than $X_{0}$ and $Y_{0}$ are homeomorphic.

Proof. Let $\left(U_{n}\right)_{n \in N}$ and $\left(V_{n}\right)_{n \in N}$ be open covers of $X$ and $Y$, respectively, that possess the properties stated in (1.2). Let $U_{0} \equiv \varnothing \equiv V_{0}$. Let $X_{n} \equiv\left(U_{n}\right.$ $\left.U_{n-1}\right)_{0}$ and let $Y_{n} \equiv\left(V_{n}-V_{n-1}\right)_{0}$, for each $n \in N$. $X_{n}$ and $Y_{n}$ are each of power the continuum and each has the cofinite topology (1.1); thus there exists a bijection $h_{n}$ of $X_{n}$ onto $Y_{n}$, and $h_{n}$ is a homeomorphism. Clearly $\left(X_{n}\right)_{n \in N}$ partitions $X$ and $\left(Y_{n}\right)_{n \in N}$ partitions $Y$. Let $h \equiv \cup_{n \in N} h_{n}$; then $h$ injects $X$ onto $Y$. Let $D^{\prime} \in$ prop $\Delta(Y)$ and let $D \equiv h^{-1}\left(D^{\prime}\right)$. Since each $D^{\prime} \cap Y_{n}$ is finite, each $D \cap X_{n}$ is finite, hence $D \in$ prop $\Delta(X)$, proving that $h$ is continuous. Similarly one sees that $h^{-1}$ is continuous, proving the theorem.

By analogy with the idea of a filter of sets, a nonempty family $\delta$ of $\Delta$ is called a $\Delta$-filter [1] if the following hold:

(1.a) $\varnothing \notin \delta$,

(1.b) $U$ and $V$ in $\delta$ implies $U \cap V \in \delta$,

(1.c) $U \in \delta$ and $W \in \Delta$ such that $U \subset W$, implies $W \in \delta$.

(Cf. the notion of $z$-filter in Hewitt [13] and in Gillman and Jerison [8].) The following useful lemma and its application to maximal ideals evolved gradually in the work of Helmer [10], Hewitt [13], Henriksen [11], [12], Gillman and Jerison [8], and the author [1].

Lemma 1.4. Given $I \in$ prop $I(A), Z(I)$ is a $\Delta$-filter. Given a $\Delta$-filter $\delta$ then $Z^{-1}(\delta)(\equiv\{f \in A: Z(f) \in \delta\})$ is in prop $I(A) . I \subset Z^{-1} Z(I)$; thus $I$ is maximal if and only if $Z(I)$ is a maximal $\Delta$-filter.

Maximal $\Delta$-filters are called $\Delta$-ultrafilters [1]. Let $\delta X$ denote the set of all $\Delta$-ultrafilters. Given $D \in \Delta$, let $\operatorname{cl}_{\delta X} D \equiv\{\mu \in \delta X: D \in \mu\}$, and let $\delta X$ have the topology for which $\left\{\operatorname{cl}_{\delta x} D: D \in \Delta\right\}$ is a basis for the closed sets. Given $x \in X$ let $\mu_{x} \equiv\{D \in \Delta: x \in D\} \equiv d(x)$; then $d$ is an injection of $X$ into $\delta X$. 
A $\Delta$-filter $\delta$ is called free or fixed according as $\cap_{D \in \delta} D$ is empty or nonempty. $I \in$ prop $I(A)$ is called free or fixed according as $Z(I)$ is free or fixed. It will be convenient to call $A$ a free ideal in $A$. Then, following Hewitt's terminology [13], $I \in I(A)$ is fixed if and only if there exists $x \in X$ such that $I \subset M_{x}$; and $I$ is free if and only if it is not fixed. Given $D \in \Delta$, $\left(\mathrm{cl}_{\delta X} D\right) \cap d(X)=d(D)$; thus $d$ is a homeomorphism of $X_{0}$ onto $d(X)$. It is frequently convenient to regard $d$ as an identification and thus regard $X_{0}$ as a subspace of $\delta X$. Clearly $Z\left(M_{x}\right)=\mu_{x}$. Given $\mu \in \delta X$ let $M_{\mu} \equiv Z^{-1}(\mu)$. Let $D_{1}$ be a nonempty element of prop $\Delta$ and let $\delta$ be a $\Delta$-filter such that $D_{1} \in \delta$. Let $\delta \mid D_{1} \equiv\left\{D \cap D_{1}: D \in \delta\right\}$; then $\delta \mid D_{1}$ is a filter on $D_{1}$. If $\delta \subset \delta^{\prime}, \delta^{\prime}$ a $\Delta$-filter, then clearly $\delta\left|D_{1} \subset \delta^{\prime}\right| D_{1}$. Given a filter $\varphi$ on $D_{1}$, let ext $\varphi$, the extension of $\varphi$, be $\left\{D \in \Delta: D \cap D_{1} \in \varphi\right\}$; then ext $\varphi$ is a $\Delta$-filter. Clearly $\operatorname{ext}\left(\delta \mid D_{1}\right)=\delta$ and $(\operatorname{ext} \varphi) \mid D_{1}=\varphi$. Given any filter $\varphi^{\prime}$ for which $\varphi \subset \varphi^{\prime}$, then ext $\varphi \subset$ ext $\varphi^{\prime}$. Let $\beta D_{1}$ denote the set of all ultrafilters on $D_{1}$. Given $S \subset D_{1}$ let $\mathrm{cl}_{\beta D_{1}} S \equiv\left\{\rho \in \beta D_{1}: S \in \rho\right\}$ and let $\left\{\operatorname{cl}_{\beta D_{1}} S: S \subset D_{1}\right\}$ serve as a basis for the closed sets in $\beta D_{1}$. Then $\beta D_{1}$ is the Stone-Cech compactification of $D_{1}$. (See e.g. [8] for details.) It is frequently convenient to identify $x \in D_{1}$ with $\rho_{x}$, $\rho_{x} \equiv\left\{S \subset D_{1}: x \in S\right\} \in \beta D_{1}$. Note that $x \in D_{1} \mapsto \rho_{x} \in \beta D_{1}$ is a homeomorphism of $D_{1}$ onto a dense subset of $\beta D_{1}$. Finally, note that if $D_{1}$ is finite $D_{1}=\beta D_{1}$, and if $D_{1}$ is infinite-and hence countable- $\beta D_{1}$ is homeomorphic to $\beta N$.

LEMMA 1.5. ext is a homeomorphism of $\beta D_{1}$ onto $\mathrm{cl}_{\delta X} D_{1}$, and $\mu \in \operatorname{cl}_{\delta X} D_{1} \mapsto$ $\mu \mid D_{1} \in \beta D_{1}$ is its inverse.

Proof. Let $\rho \in \beta D_{1}$, let $\mu \equiv \operatorname{ext} \rho$, and let $\mu \subset \mu^{\prime} \in \delta X$; then $\rho \subset \rho^{\prime} \equiv$ $\mu^{\prime} \mid D_{1}$. Since $\rho$ is maximal, $\rho=\rho^{\prime}$, and so $\mu=\mu^{\prime}$, proving that ext maps $\beta D_{1}$ into $\mathrm{cl}_{\delta X} D_{1}$. A similar argument shows that if $\mu \in \mathrm{cl}_{\delta X} D_{1}$, then $\mu \mid D_{1} \in \beta D_{1}$. As noted above, ext and $\mu \mapsto \mu \mid D_{1}$ are inverses to each other. Let $S \subset D_{1}$. Since $\operatorname{ext}\left(\operatorname{cl}_{\beta D_{1}} S\right)=\operatorname{cl}_{\delta X} S$, ext is a homeomorphism, proving the lemma.

THEOREM 1.6. $Z: M \in \operatorname{specm} A(X) \mapsto Z(M) \in \delta X$ is a homeomorphism of specm $A(X)$ onto $\delta X$; thus $\delta X$ is a compact $T_{1}$-space, which is not $T_{2}$.

Proof. Applying (1.4) we see that $Z$ is a bijection. Let $f \in A$ and let $D \equiv Z(f)$. Then $f \in M$ if and only if $D \in Z(M)$. Clearly $Z(V((f)))=$ $\mathrm{cl}_{\delta X} D$; thus $Z$ is a homeomorphism.

THEOREM 1.7. Let $h$ be a homeomorphism of $X_{0}$ onto $Y_{0}$. $h$ extends to a unique homeomorphism $H$ of $\delta X$ onto $\delta Y$.

Proof. $h(\Delta(X))=\Delta(Y)$; thus $h$ induces an injection $H$ of $\delta X$ onto $\delta Y$. Let $D \in \Delta(X)$. Clearly $H\left(\mathrm{cl}_{\delta X} D\right)=\mathrm{cl}_{\delta Y} h(D)$, proving that $H$ is a homeomorphism. Since $X_{0}$ is dense in $\delta X$ and $Y_{0}$ is dense in $\delta Y, H$ is unique. 
THEOREM 1.8. Given any noncompact (connected) Riemann surfaces $X$ and $Y$, then specm $A(X)$ and specm $A(Y)$ are homeomorphic.

This follows from (1.3), (1.6), and (1.7).

2. Divisors and ideals. The following can easily be deduced from Florack's Theorem.

LEMMA 2.1. For each $f \in F^{*}(\equiv F-\{0\})$ there exist $a$ and $b$ in $A-\{0\}$ such that $f=a / b$ and $(a, b)=1$. Further, $a$ and $b$ are unique up to multiplication by $a$ unit in $A$.

Given $f \in F^{*}$ and $x \in X$, let $x_{x} \nu_{x}(f)$ (or $\nu_{x}(f)$ for short) be the order of $f$ at $x$ in $X$; then $\nu_{x}(0) \equiv \infty_{x}$, an element defined to be greater than every integer. Let $n+\infty_{x} \equiv \infty_{x} \equiv \infty_{x}+n$, for all integers $n . \nu_{x}$ is then a discrete rank one valuation on $F$ over C. (See e.g. [20] for a general reference on valuation theory, or better still, see Krull's classic [17].) (Iss'sa's Theorem [14] shows that any such valuation is of this form.) The valuation ring of $\nu_{x}$ is $A_{M_{x}}$ ( $\equiv\left\{a / b: a \in A\right.$ and $\left.b \in A-M_{x}\right\}$ ). In passing, note that $A=\cap_{x \in X} A_{M_{x}}$; thus $A$ is the ring of "integers" of $F$. Let $Z^{X}$ denote the set of all maps of $X$ into $Z$, the addition group of integers. Under pointwise operations $Z^{X}$ is, of course, a lattice ordered group. For $a \in Z^{X}$ let the support of $a, \operatorname{supp}(a)$, be $\{x \in X: a(x) \neq 0\}$. Let $\operatorname{Div} X$, the divisors on $X$, be $\left\{a \in Z^{X}: \operatorname{supp}(a)\right.$ is a proper closed subset of $X_{0}$ \}. Clearly Div $X$ is a lattice ordered subgroup of $Z^{X}$ that is completely determined by the topology on $X_{0}$. Let $f \in F^{*}$ and let

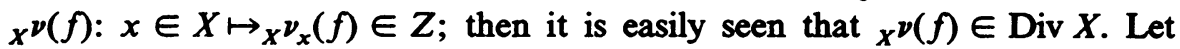
$U(X)$ (or $U$ for short) denote the group of units of $A(X)$. Clearly $U=\{f \in$ $A: Z(f)=\varnothing\}$. From Florack's Theorem we deduce:

THEOREM 2.2. The following sequence on the category of abelian groups is exact:

$$
0 \rightarrow U(X) \hookrightarrow F^{*}(X) \stackrel{x^{\nu}}{\rightarrow} \operatorname{Div} X \rightarrow 0 .
$$

When no confusion will arise let $\nu \equiv x^{\nu}$. Let $h$ be a homeomorphism of $X_{0}$ onto $Y_{0}$. Given $b \in \operatorname{Div} Y$, let $h^{*}(b) \equiv b \circ h$; then we have:

THEOREM 2.3. $h^{*}$ is a lattice-preserving group isomorphism of Div $Y$ onto Div $X$; thus $\operatorname{Div} Y$ and $\operatorname{Div} X$ are always isomorphic.

Let $\infty$ denote an element greater than every element of Div $X$, and let $\nu(0) \equiv \infty$. Further, let $a+\infty \equiv \infty \equiv \infty+a$, for all $a \in(\operatorname{Div} X) \cup\{\infty\}$.

THEOREM 2.4. Given $f, g \in F, \nu(f g)=\nu(f)+\nu(g)$, and $\nu(f \pm g)>\nu(f) \wedge$ $\nu(g)$. Let

$$
X_{1} \equiv\left\{x \in X: \nu_{x}(f) \neq \nu_{x}(g)\right\}
$$

then $\nu(f \pm g)\left|X_{1}=(\nu(f) \wedge \nu(g))\right| X_{1}$. 
Let $\operatorname{Div}^{+} X \equiv\{a \in \operatorname{Div} X: a>0\}$. Clearly $A-\{0\}=\nu^{-1}\left(\operatorname{Div}^{+} X\right)$. Let $f \in A-\{0\}$ and let $g \in A . g$ divides $f$ if and only if $\nu(g)<\nu(f)$; thus divisibility properties in $A$ are faithfully represented in $\operatorname{Div}^{+} X$. Let $f_{1}, \ldots, f_{n}$ $\in A$, at least one of which is nonzero. Since $\operatorname{Div}^{+} X$ is a lattice, $\nu\left(f_{1}\right)$ $\wedge \cdots \wedge \nu\left(f_{n}\right) \equiv a$ is in $\operatorname{Div}^{+} X$. By (2.2), there exists $g \in A$ such that $\nu(g)=a$; thus $g\left|f_{1}, \ldots, g\right| f_{n}$. Let $h$ be a common divisor of $f_{1}, \ldots, f_{n}$ in $A$; then $\nu(h)<\nu(g)$, and so $h \mid g$, showing that $g=\operatorname{gcd}\left(f_{1}, \ldots, f_{n}\right)$.

HeLmeR's Lemma 2.5 ([10], [1]). There exists $a_{1}, \ldots, a_{n} \in A$ such that $g=a_{1} f_{1}+\cdots+a_{n} f_{n}$; hence $\left(f_{1}, \ldots, f_{n}\right)=(g)$. Thus every finitely generated ideal in $A$ is principal.

Initially the concern of the researchers in this field was with the ideal theory of $A(C)$. In 1946 Schilling [18] considered fractional ideals of $F(C)$. We will generalize this in two directions by considering ssub- $A$-modules of $F(X)\} \equiv \operatorname{sam}(F(X))$ (or $\operatorname{sam}(F)$ for short). Clearly $\operatorname{sam}(F)$ is a partially ordered set under inclusion, that is inductive, closed under intersection, and has a least element 0 and a greatest element $F . I \in \operatorname{sam}(F)$ is called a fractional ideal if there exists $f \in F^{*}$ so that If $\subset A$. Let $I(F) \equiv$ fractional ideals of $F\}$. Let $I(A) \equiv$ ideals of $A\}$, and prop $I(A) \equiv I(A)-\{A\}$. Clearly

$$
\text { prop } I(A) \varsubsetneqq I(A) \varsubsetneqq I(F) \varsubsetneqq \operatorname{sam}(F) .
$$

Each of these partially ordered set of sets is closed under intersection and, with the exception of $I(F)$, each is inductive.

(Div $X) \cup\{\infty\}$ is a lattice. A dual ideal [0] $J$ in this lattice is a nonempty subset of it such that the following hold.

(2a) Given $j \in J$ and $a \in(\operatorname{Div} X) \cup\{\infty\}$ such that $j<a$; then $a \in J$;

(2b) $J$ is closed under finite $\wedge$.

Let $\operatorname{di}(\operatorname{Div} X) \equiv\{$ dual ideals of $(\operatorname{Div} X) \cup\{\infty\}$.

Let $J(\operatorname{Div} X) \equiv\{J \in \operatorname{di}(\operatorname{Div} X):$ there exists $d \in \operatorname{Div} X$ such that $d<j$, for all $j \in J\}$, let $J\left(\operatorname{Div}^{+} X\right) \equiv\{J \in \operatorname{di}(\operatorname{Div} X): 0<j$ for all $j \in J\}$, and let

Clearly

$$
\text { prop } \left.J\left(\operatorname{Div}^{+} X\right) \equiv J\left(\operatorname{Div}^{+} X\right)-\left\{\operatorname{Div}^{+} X\right) \cup\{\infty\}\right\} \text {. }
$$

$$
\operatorname{prop} J\left(\operatorname{Div}^{+} X\right) \varsubsetneqq J\left(\operatorname{Div}^{+} X\right) \varsubsetneqq J(\operatorname{Div} X) \varsubsetneqq \operatorname{di}(\operatorname{Div} X),
$$

and clearly each of these sets is partially ordered under inclusion, each is closed under intersection, and, with the exception of $J(\operatorname{Div} X)$, each is inductive.

Theorem 2.6. Let $I \in \operatorname{sam}(F)$, then $\nu(I)$ is in $\operatorname{di}(\operatorname{Div} X)$. Given $J \in$ $\operatorname{di}(\operatorname{Div} X), \nu^{-1}(J) \in \operatorname{sam}(F)$, and $I=\nu^{-1} \nu(I)$. (Of course $J=\nu \nu^{-1}(J)$ ) Thus $\nu$ is an injection of $\operatorname{sam}(F)$ onto $\operatorname{di}(\operatorname{Div} X)$. I is in $I(F), I(A)$, or prop $I(A)$, if and only if $\nu(I)$ is in $J(\operatorname{Div} X), J\left(\operatorname{Div}^{+} X\right)$, or prop $J\left(\operatorname{Div}^{+} X\right)$, respectively. 
Proof. Let $I \in \operatorname{sam}(F)$ and let $J \equiv \nu(I)$. Let $j \in J$ and let $a \in(\operatorname{Div} X) \cup$ $\{\infty\}$ such that $j<a$. Since $j \in J$ there exists $i \in I$ such that $\nu(i)=j$. By (2.2) there exists $f \in F$ for which $\nu(f)=a$. If $j=\infty$ then so is $a$, and hence $a \in J$. Assume that $j<\infty$; then $i \neq 0$. Since $\nu(f / i)=a-j>0, f / i \in A$. Since $I$ is an $A$-module, $f=(f / i) i$ is in $I$ : thus $a \in J$, proving that $J$ satisfies condition (2a). Let $j^{\prime} \in J$. If $j$ or $j^{\prime}$ is $\infty$ then $j \wedge j^{\prime}$ is $j^{\prime}$ or $j$, respectively, and hence $j \wedge j^{\prime}$ is in $J$. Assume that $j \neq \infty \neq j^{\prime}$. There exists $i^{\prime} \in I$ such that $\nu\left(i^{\prime}\right)=j^{\prime}$. Since $j \wedge j^{\prime}<\infty$, there exists $f \in F^{*}$ for which $\nu(f)=j \wedge j^{\prime}$; thus $i / f$ and $i^{\prime} / f$ are in $A$. Since $\nu(f)=\nu(i) \wedge \nu\left(i^{\prime}\right)$,

$$
\begin{aligned}
\nu(i / f) \wedge \nu\left(i^{\prime} / f\right) & =(\nu(i)-\nu(f)) \wedge\left(\nu\left(i^{\prime}\right)-\nu(f)\right) \\
& =\left(\nu(i) \wedge \nu\left(i^{\prime}\right)\right)-\nu(f)=0=\nu(1)
\end{aligned}
$$

thus $\left(i / f, i^{\prime} / f\right)=1$. By Helmer's Lemma (2.5), there exist $h, k \in A$ such that $1=h i / f+k i^{\prime} / f$, i.e., $f=h i+k i^{\prime}$. Since $I$ is an $A$-module, $f$ is in $I$, and so $j \wedge j^{\prime}=\nu(f) \in J$, proving that $J$ satisfies (2b), and so $J$ is in $\operatorname{di}(\operatorname{Div} X)$. Now let $J^{\prime}$ be in $\operatorname{di}(\operatorname{Div} X)$ and let $I^{\prime} \equiv \nu^{-1}\left(J^{\prime}\right)$. Since $J^{\prime} \neq \varnothing$ and since $\nu$ maps $F$ onto $(\operatorname{Div} X) \cup\{\infty\}, I^{\prime} \neq \varnothing$. Let $i, i^{\prime} \in I^{\prime}$ and let $f \in A . \nu(f i)=\nu(f)+\nu(i)$ $>\nu(i)$, showing that $f i \in I^{\prime}$. By (2.4), $\nu\left(i-i^{\prime}\right)>\nu(i) \wedge \nu\left(i^{\prime}\right)$, showing that $i-i^{\prime} \in I^{\prime}$, and hence that $I^{\prime}$ is in $\operatorname{sam}(F)$. Since $U$, the group of units of $A$, is the kernel of $\nu$ (2.2), given any $I \in \operatorname{sam}(F), I=\nu^{-1} \nu(I)$. The rest is obvious.

Banaschewski [4] proved that $\nu$ induces an order preserving bijection between $I(A(C))$ and $J\left(\mathrm{Div}^{+} \mathrm{C}\right)$, in 1958; thus (2.6) is a generalization of his result in two ways. Following Banaschewski let maximal elements in prop $J\left(\mathrm{Div}^{+} X\right)$ be called maximal dual ideals in $\mathrm{Div}^{+} X . J \in$ prop $J\left(\mathrm{Div}^{+} X\right)$ will be called a prime (resp., primary) dual ideal in $\operatorname{Div}^{+} X$ if given any $a$, $b \in \operatorname{Div}^{+} X$ such that $a+b \in J$, then $a$ or $b$ is in $J$ (resp., $a+b \in J$ and $a \notin J$ implies the existence of $n \in N$ such that $n b \in J$ ).

BANASCHEWSKI's THEOREM 2.7 [4]. $I \in I(A)$ is maximal, prime, or primary according as $J \equiv \nu(I)$ is a maximal, prime, or primary dual ideal in $\mathrm{Div}^{+} X$.

On the strength of his extensive analysis, Banaschewski concluded at the end of [4, p. 160] that, "The multiplicative ideal theory of $A$ is hence identical with the dual ideal theory of $\mathrm{Div}^{+} X$ " (author's translation and notation). Generalizing his analysis still further let us consider all the classical ideal theoretic operations on $\operatorname{sam}(F)$. Given $I$ and $I^{\prime}$ in $\operatorname{sam}(F)$, it is easy to see that $I+I^{\prime}, I \cap I^{\prime}, I \cdot I^{\prime}$, and $I: I^{\prime}\left(\equiv\left\{f \in F: f I^{\prime} \subset I\right\}\right)$ are again in $\operatorname{sam}(F)$. It is easy to see that $I(F)$ is also closed under $+, \cap, \cdot$, and $:$, and that $I(A)$ is closed under,$+ \cap$, and - . (See e.g. [19] for a general reference on classical ideal theory.) We will define analogous operations for di(Div $X$ ).

Let $J$ and $J^{\prime}$ be in $\operatorname{di}(\operatorname{Div} X)$. Let $J \wedge J^{\prime} \equiv\left\{j \wedge j^{\prime}: j \in J\right.$ and $\left.j^{\prime} \in J^{\prime}\right\}$. 
Lemma 2.8. $J \wedge J^{\prime}$ is in $\operatorname{di}(\operatorname{Div} X)$. If $J$ and $J^{\prime}$ are both in $J(\operatorname{Div} X)$ and $J\left(\operatorname{Div}^{+} X\right)$, respectively then so is $J \wedge J^{\prime}$.

Proof. Let $J^{\prime \prime} \equiv J \wedge J^{\prime}$, let $j \in J$, and let $j^{\prime} \in J^{\prime}$, with $j^{\prime \prime} \equiv j \wedge j^{\prime}$. Let $b \in(\operatorname{Div} X) \cup\{\infty\}$ such that $b \geqslant j^{\prime \prime}$. Since $b \vee j>j$ and $b \vee j^{\prime}>j^{\prime}, b \vee j$ $\in J$ and $b \vee j^{\prime} \in J^{\prime}$. Since $b=(b \vee j) \wedge\left(b \vee j^{\prime}\right), b \in J^{\prime \prime}$. Clearly $J^{\prime \prime}$ is closed under finite $\wedge$, proving that $J^{\prime \prime}$ is in di(Div $X$ ). The rest of the lemma is clear.

LEMMA 2.9. Let $I$ and $I^{\prime}$ be in $\operatorname{sam}(F)$; then $\nu\left(I+I^{\prime}\right)=\nu(I) \wedge \nu\left(I^{\prime}\right)$.

Proof. Let $i \in I, i^{\prime} \in I^{\prime}, j \equiv \nu(i), j^{\prime} \equiv \nu\left(i^{\prime}\right)$, and $j^{\prime \prime} \equiv \nu\left(i-i^{\prime}\right)$. By (2.4), $j^{\prime \prime}>j \wedge j^{\prime}$; thus $\nu\left(I+I^{\prime}\right) \subset \nu(I) \wedge \nu\left(I^{\prime}\right)$. To prove the lemma, it suffices to prove that $j \wedge j^{\prime}$ is in $\nu\left(I+I^{\prime}\right)$. If $i$ or $i^{\prime}$ is zero, then $j \wedge j^{\prime}$ is equal to $j^{\prime}$ or $j$, respectively, and hence is in $\nu\left(I+I^{\prime}\right)$. Assume that $i \neq 0 \neq i^{\prime}$. Let $D \equiv$ $\operatorname{supp}(j), D^{\prime} \equiv \operatorname{supp}\left(j^{\prime}\right)$, and $D^{\prime \prime} \equiv \operatorname{supp}\left(j^{\prime \prime}\right)$; then $D, D^{\prime}$, and $D^{\prime \prime}$ are in prop $\Delta$. Clearly $D^{\prime \prime} \subset D \cup D^{\prime} \equiv E$, and $E$ is in prop $\Delta$. (Note: to show that a subset $S$ of $X$ is in prop $\Delta$ it is necessary and sufficient that $S \cap U_{n}$ be finite, for all $n \in N,\left(U_{n}\right)_{n \in N}$ being as described in (1.2).) Let

$$
E_{1} \equiv\left\{x \in E: j^{\prime \prime}(x)>j(x) \wedge j^{\prime}(x)\right\}
$$

and let $E_{2} \equiv E-E_{1}$. Then $E_{1}$ and $E_{2}$ are in prop $\Delta$, and these sets partition $E$. Using a corollary of Florack's Theorem [1, (1.4)], we know that there exists $b \in A(X)$ such that $b \mid E_{2}=0$ and $b \mid E_{1}=1$. Let $a=e^{b}$; then $a \in U$ and so $\nu(a)=0$. Let $g^{\prime} \equiv a i^{\prime}$ and note that, since $I^{\prime}$ is an $A$-module, $g^{\prime} \in I^{\prime}$. Clearly $\nu\left(g^{\prime}\right)=\nu(a)+\nu\left(i^{\prime}\right)=\nu\left(i^{\prime}\right)=j^{\prime} ;$ thus supp $\nu\left(g^{\prime}\right)=\operatorname{supp}\left(j^{\prime}\right)=D^{\prime} \subset E$ and so supp $\nu\left(i-g^{\prime}\right) \subset E$. Let $x \in X$ and let $t_{x}$ be a local uniformizer at $x$. The Laurent expansions of $i$ and $i^{\prime}$ at $x$ begin with $c_{x} t_{x}^{m_{x}}$ and $c_{x}^{\prime} t_{x}^{m_{x}^{\prime}}$, respectively, where $c_{x}$ and $c_{x}^{\prime}$ are in $C$, and $m_{x}$ and $m_{x}^{\prime}$ are integers. If $m_{x} \neq m_{x}^{\prime}$, then $j^{\prime \prime}(x)=j(x) \wedge j^{\prime}(x)$; thus $x \in E_{2}$. If $m_{x}=m_{x}^{\prime}$ and $c_{x} \neq c_{x}^{\prime}$, then $j^{\prime \prime}(x)=j(x)$ $\wedge j^{\prime}(x)$, and hence $x \in E_{2}$. Thus we see that if $x \in E_{1}$, then $m_{x}=m_{x}^{\prime}$ and $c_{x}=c_{x}^{\prime}$. If $x$ is in $E_{2}$, then the Laurent series for $i^{\prime}$ and $g^{\prime}$ at $x$ each begins with $c_{x}^{\prime} t_{x}^{m_{x}^{\prime}}$; and

$$
\nu\left(i-g^{\prime}\right)\left|E_{2}=\nu\left(i-i^{\prime}\right)\right| E_{2} .
$$

If $x \in E_{1}$, then the Laurent series for $i$ and $g^{\prime}$ at $x$ begin with $c_{x} t_{x}^{m_{x}}$ and $e c_{x} t_{x}^{m_{x}}$, respectively; thus $\nu_{x}\left(i-g^{\prime}\right)=m_{x}$ and hence $\nu\left(i-g^{\prime}\right)\left|E_{1}=j \wedge j^{\prime}\right| E_{1}$. Since $\operatorname{supp}\left(\nu\left(i-g^{\prime}\right)\right)$ and $\operatorname{supp}\left(j \wedge j^{\prime}\right)$ are each subsets of $E$, we have proved that $\nu\left(i-g^{\prime}\right)=j \wedge j^{\prime}$, proving that $j \wedge j^{\prime}$ is in $\nu\left(I+I^{\prime}\right)$, and proving the lemma.

Let $J$ and $J^{\prime}$ be in $\operatorname{di}(\operatorname{Div} X)$. Let $J+J^{\prime}$ be defined to be the set of all $\wedge_{k=1}^{n}\left(j_{k}+j_{k}^{\prime}\right)$, where $j_{k} \in J$ and $j_{k}^{\prime} \in J^{\prime}$, and $n \in N$. 
LemMa 2.10. $J+J^{\prime}$ is in $\operatorname{di}(\operatorname{Div} X)$. If $J, J^{\prime} \in J(\operatorname{Div} X)\left(\operatorname{resp} ., J\left(\operatorname{Div}^{+} X\right)\right)$, then $J+J$ is in $J(\operatorname{Div} X)$ (resp., $J\left(\operatorname{Div}^{+} X\right)$ ). Given $I, I^{\prime} \in \operatorname{sam}(F)$, then $\nu\left(I \cdot I^{\prime}\right)=\nu(I)+\nu\left(I^{\prime}\right)$.

Proof. Clearly $J+J^{\prime} \equiv J^{\prime \prime}$ is closed under finite $\wedge$. Now let $b \in(\operatorname{Div} X)$ $\cup\{\infty\}$ such that $(n): b>\wedge_{k=1}^{n}\left(j_{k}+j_{k}^{\prime}\right)$. If $b=\infty$, then $b=\infty+\infty$. Without loss of generality we may assume that each $j_{k}<\infty$, and each $j_{k}^{\prime}<\infty$, in condition ( $n$ ) above. Assume first that $n=1$; then $b>j_{1}+j_{1}^{\prime}$, and so $b-j_{1}^{\prime} \geqslant j_{1}$; hence $b-j_{1}^{\prime} \in J$, and we conclude that $b \in J^{\prime \prime}$. For general $n \in N$,

$$
b=b \bigvee\left(\bigwedge_{k=1}^{n}\left(j_{k}+j_{k}^{\prime}\right)\right)
$$

which, since Div $X$ is a distribution lattice, is $\bigwedge_{k=1}^{n}\left(b \vee\left(j_{k}+j_{k}^{\prime}\right)\right)$. Since

$$
b \vee\left(j_{k}+j_{k}^{\prime}\right) \geqslant j_{k}+j_{k}^{\prime} \in J^{\prime \prime}
$$

we may use the result above, in case $n=1$, to conclude that $b \vee\left(j_{k}+j_{k}^{\prime}\right)$ is in $J^{\prime \prime}$ for each $k$. Since $J^{\prime \prime}$ is closed under finite $\wedge, b \in J^{\prime \prime}$, proving that $J^{\prime \prime} \in \operatorname{di}(\operatorname{Div} X)$. Assume further that $J, J^{\prime} \in J(\operatorname{Div} X)$. By definition there exists $d, d^{\prime} \in \operatorname{Div} X$ so that $d<j$ and $d^{\prime}<j^{\prime}$, for all $j \in J$ and all $j^{\prime} \in J^{\prime}$. Clearly $d+d^{\prime}<j^{\prime \prime}$ for all $j^{\prime \prime} \in J^{\prime \prime}$, showing that $J^{\prime \prime} \in J(\operatorname{Div} X)$. If $J$ and $J^{\prime}$ are in $J\left(\operatorname{Div}^{+} X\right)$ we may take $d$ and $d^{\prime}$ above to be zero; thus $J+J^{\prime} \in$ $J\left(\mathrm{Div}^{+} X\right)$. Let $i_{k} \in I$ and $i_{k}^{\prime} \in I^{\prime} . I \cdot I^{\prime}$, of course, consists of all elements of the form $x=\sum_{k-1}^{n} i_{k} i_{k}^{\prime}$. By (2.4), $\nu(x)>\bigwedge_{k=1}^{n}\left(\nu\left(i_{k}\right)+\nu\left(i_{k}^{\prime}\right)\right)$; thus $\nu\left(I \cdot I^{\prime}\right) \subset$ $\nu(I)+\nu\left(I^{\prime}\right)$. Since $\nu(I)+\nu\left(I^{\prime}\right)$ is generated, using $\wedge$, by all the elements of the form $\nu\left(i_{k}\right)+\nu\left(i_{k}^{\prime}\right)$, each of which is in $\nu\left(I \cdot I^{\prime}\right)$, and since $\nu\left(I \cdot I^{\prime}\right)$ is closed under finite $\wedge$, we see that $\nu(I)+\nu\left(I^{\prime}\right) \subset \nu\left(I^{\prime} \cdot I^{\prime}\right)$, proving that $\nu\left(I^{\prime} \cdot I^{\prime}\right)=$ $\nu(I)+\nu\left(I^{\prime}\right)$; completing the proof of the lemma.

Recall that a nonempty subset $S$ of $A-\{0\}$ that is closed under multiplication is called a multiplicative system in $A$. Let $I \in \operatorname{sam}(F)$ and let $S^{-1} I \equiv\{i / s: i \in I$ and $s \in S\}$; then $S^{-1} I$ is again in $\operatorname{sam}(F)$. Note that $S^{-1} I=I \cdot\left(S^{-1} A\right)$. If $1 \in S$ then $I \subset S^{-1} I$. Let $P \in \operatorname{spec} A$ and let $S \equiv A$ - $P$; then $S$ is a multiplicative system in $A$ which contains 1 . In this case $S^{-1} I$ is usually denoted by $I_{P}$. A nonempty subset $T$ of $\mathrm{Div}^{+} X$ that is closed under addition will be called an additive system in $\operatorname{Div}^{+} X$. Let $T$ be an additive system in $\operatorname{Div}^{+} X$, let $J \in \operatorname{di}(\operatorname{Div} X)$, and let $J(-T) \equiv\{j-t: j \in J$ and $t \in T$ \}. If $0 \in T$, then $J \subset J(-T)$. Given a prime dual ideal $Q$ in $\left(\mathrm{Div}^{+} X\right) \cup\{\infty\}$, then $\operatorname{Div}^{+} X-Q \equiv T$ is an additive system in $\operatorname{Div}^{+} X$ which contains 0 . In this case $J(-T)$ will frequently be denoted by $J_{Q}$.

LEMMA 2.11. Let $S$ be a nonempty subset of $A-\{0\}$ and let $T \equiv \nu(S)$. Let $I \in \operatorname{sam}(F)$ and let $J \equiv \nu(I)$. Let $P \in \operatorname{spec} A$ and let $Q \equiv \nu(P)$.

(1) $S$ is a multiplicative system of $A$ if and only if $T$ is an additive system of $\operatorname{Div}^{+} X$; 
(2) $1 \in S$ if and only if $0 \in T$;

(3) $\nu\left(S^{-1} I\right)=J(-T)$;

(4) Thus, $J(-T)$ is in $\operatorname{di}(\operatorname{Div} X)$.

(5) $\nu\left(I_{P}\right)=J_{Q}$

Proof. (1), (2), (3), and (5) are obvious. (3), the fact that $S^{-1} I \in \operatorname{sam}(F)$, and (2.6) imply (4).

Let $I$ and $I^{\prime}$ be in $\operatorname{sam}(F)$. Recall that the quotient module $I: I^{\prime}$ is $\left\{f \in F: f I^{\prime} \subset I\right\}$. Let $J, J^{\prime} \in \operatorname{di}(\operatorname{Div} X)$ and let $J-J^{\prime} \equiv\{a \in(\operatorname{Div} X) \cup$ $\left.\{\infty\}: a+J^{\prime} \subset J\right\}$.

LEMMA 2.12. $J-J^{\prime}$ is in $\operatorname{di}(\operatorname{Div} X)$, and $\nu\left(I: I^{\prime}\right)=\nu(I)-\nu\left(I^{\prime}\right)$.

Proof. Let $a \in J-J^{\prime}$, i.e., $a+j^{\prime} \in J$ for all $j^{\prime} \in J$. Let $b \in(\operatorname{Div} X) \cup$ $\{\infty\}$, with $b>a$ : then $b+j^{\prime} \geqslant a+j^{\prime}$ and hence $b+j^{\prime} \in J$, for all $j^{\prime} \in J^{\prime}$; proving that $b$ is in $J-J^{\prime}$. Let $c \in J-J^{\prime}$. We wish to show that $a \wedge c$ is in $J-J^{\prime}$. If $c=\infty$, then $a \wedge c=a$, which is in $J-J^{\prime}$. Assume that $a, c$, and $j^{\prime}$ are all in Div $X$. Since Div $X$ is a lattice ordered group,

$$
(a \wedge c)+j^{\prime}=\left(a+j^{\prime}\right) \wedge\left(c+j^{\prime}\right) \text {. }
$$

Since $a+j^{\prime}$ and $c+j^{\prime}$ are in $J,\left(a+j^{\prime}\right) \wedge\left(c+j^{\prime}\right)$ is in $J$; and so $(a \wedge c)+$ $j^{\prime} \in J$. Thus $J-J^{\prime}$ is in $\operatorname{di}(\operatorname{Div} X)$. Clearly $\nu\left(I: I^{\prime}\right)=\nu(I)-\nu\left(I^{\prime}\right)$, proving the lemma.

By (1.3) there exists a homeomorphism $h$ of $X_{0}$ onto $Y_{0}$. By (2.3), $h$ engenders a lattice preserving group isomorphism $h^{*}$ of Div $Y$ onto Div $X$. Let $h^{*}$ map the infinite divisor over $Y$ to the infinite divisor over $X$; then $h^{*}$ induces an order preserving injection of $\operatorname{di}(\operatorname{Div} Y$ ) onto $\operatorname{di}(\operatorname{Div} X)$, which preserves $\wedge, \cap,+,-$. From (2.6), (2.7), (2.9), (2.10) and (2.12) we obtain the following main theorem.

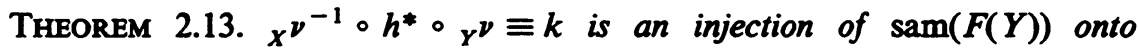
$\operatorname{sam}(F(X))$ that preserves inclusion, $+, \cap, \cdot$, and $:$, maps $I(F(Y))$ onto $I(F(X))$ and $I(A(Y)$ ) onto $I(A(X))$, and takes maximal, prime and primary ideals in $A(Y)$ to ideals of the same sort in $A(X)$.

As a corollary we have:

THEOREM 2.14. $k \mid \operatorname{spec} A(Y)$ is a homeomorphism onto spec $A(X)$.

3. Local ideal theory. An ideal $I$ in $A(X)$ will be called local if it is contained in a unique $M \in \operatorname{specm} A$, i.e., if $V(I)$ consists of a single point. Clearly each $M \in \operatorname{specm} A$ is local. Clearly 0 and $A$ are not local. Henriksen [12] proved that each nonzero $P$ in $\operatorname{spec} A(C)$ is local; thus by (1.3) or by using Henriksen's argument over $X$, where it also works, we see that every nonzero proper primary ideal in $A(X)$ is local. The following-assuredly well known-result was noted in [2, (5.2)]. 
THEOREM 3.1. For all $I \in$ prop $I(A)$,

$$
I=\bigcap_{M \in V(I)}\left(I_{M} \cap A\right)=\bigcap_{M \in \operatorname{specm} A}\left(I_{M} \cap A\right) .
$$

Corollary 3.2. Let $I \in I(F)-\{0\}$. There exists $f \in F^{*}$ so that, If $\varsubsetneqq A$, then

$$
I=\bigcap_{M \in V(I f)}\left(I_{M} \cap(A / f)\right)=\bigcap_{M \in \operatorname{specm} A}\left(I_{M} \cap(A / f)\right)
$$

LEMMA 3.3. Let $I \in$ prop $I(A)$.

(1) $V(I)=\{M\}$ implies $I=I_{M} \cap A$;

(2) $A \subset I_{M}$ if and only if $M \notin V(I)$;

(3) assume that $I \neq 0$ and that $M \in V(I)$; then $I^{\prime} \equiv I_{M} \cap A$ is local, and $V\left(I^{\prime}\right)=\{M\}$.

Proof. (1) (3.1) implies $I=I_{M} \cap A$. (2) $M \notin V(I)$ implies the existence of $i \in I-M$; thus $i \in A-M$, and so $1=i / i \in I_{M}$, and hence $A \subset I_{M}$. Conversely, assume that $A \subset I_{M}$. There exist $i \in I$ and $b \in A-M$ such that $1=i / b$, i.e., $b=i$. Hence we see that $I \cap(A-M) \neq \varnothing$, and so $M \notin$ $V(I)$. (3) Let $I \neq 0$, and assume that $M \in V(I)$. Let $I^{\prime} \equiv I_{M} \cap A$. Given $i^{\prime} \in I^{\prime}$, there exist $i \in I$ and $b \in A-M$ so that $i^{\prime}=i / b$, i.e., $b i^{\prime}=i \in I \subset$ $M$. Since $b \notin M$, and since $M$ is prime, $i^{\prime}$ is in $M$, i.e., $I^{\prime} \subset M$ and so $M \in V\left(I^{\prime}\right)$. Let $M^{\prime} \in(\operatorname{specm} A)-\{M\}$. Since $I \neq 0$ there exists $i \in I-$ \{0\}. Since $M \neq M^{\prime}, Z(M) \neq Z\left(M^{\prime}\right)$; hence there exist $D \in Z(M)$ and $D^{\prime} \in Z\left(M^{\prime}\right)$ for which $D \cap D^{\prime}=\varnothing$. Since $D, D^{\prime} \in \operatorname{prop} \Delta, D \cup D^{\prime} \in$ prop $\Delta$. By Florack's Theorem, there exists $b \in A$ for which $\nu(b) \mid D=0$, $\nu(b)\left|D^{\prime}=\nu(i)\right| D^{\prime}$, and $\nu(b)=0$ on $X-\left(D \cup D^{\prime}\right)$. Since $\nu(b) \mid D=0, Z(b) \notin$ $Z(M)$ and so $b \in A-M$. Let $i^{\prime} \equiv i / b$; then $i^{\prime} \in I^{\prime} . \nu\left(i^{\prime}\right) \mid D^{\prime}=0$, thus $Z\left(i^{\prime}\right) \notin Z\left(M^{\prime}\right)$, and so $i^{\prime} \notin M^{\prime}$, proving the lemma.

Combining (3.1) and (3.3) we obtain the following local representation theorem.

THEOREM 3.4. Let $I$ be a nonzero proper ideal in $A$; then $I=\cap_{M \in V(I)}\left(I_{M}\right.$ $\cap A)$, each $I_{M} \cap A$ being a local ideal of $A$ with $V\left(I_{M} \cap A\right)=\{M\}$.

For $M \in \operatorname{specm} A$, let $I(A, M) \equiv\{I \in I(A): V(I)=\{M\}\}$. Combining 3.3(1) with some standard commutative algebra (see e.g. [19, pp. 218-227]), we obtain

Theorem 3.5. $I \in I(A, M) \mapsto I^{e} \equiv I_{M} \in I\left(A_{M}\right)-\left\{0, A_{M}\right\}$ is an order preserving bijection, whose inverse is $I^{\prime} \mapsto\left(I^{\prime}\right)^{c} \equiv I^{\prime} \cap A$. These maps preserve + , $\cap$, and $\cdot . I \mapsto I^{e}$ takes maximal, prime and primary ideals in $I(A, M)$ to ideals of the same type in $I\left(A_{M}\right)$. 
To analyse $I\left(A_{M}\right)$, we have the following slight generalization of $[1,(2.2)]$.

Lemma 3.6. Let $P$ be a nonzero (proper) prime ideal in $A$; then $A_{P}$ is a valuation ring.

Proof. Since $P \neq 0, A_{P} \neq F$. Let $f \in F-A_{P}$. By (2.1), there exist $a$ and $b$ in $A, b \neq 0$ and $(a, b)=1$, such that $f=a / b$. Since $f \notin A_{P}, b$ is in $P$. Since $P$ is proper $1 \notin P$. Since $(a, b)=1, a$ cannot be in $P$; thus $1 / f=b / a$ is in $A_{P}$, proving the lemma.

Of particular use is the fact that $A_{M}$ is a valuation ring. In Krull's classic monograph on valuation theory [17], of 1932, it was shown that the ideal theory of $A_{M}$ and the structure of its value group are in faithful correspondence. (Recently, in [3], this analysis was extended to primary ideals of $A_{M}$.) Thus to learn about the structure of $I(A, M)$, let us compute the value group of $A_{M}$. (See e.g. [20, p. 40] for details on Krull's Theorem.) $Z(M) \equiv \mu \in \delta X$ is a directed subset of $X$. Let $G_{\mu}$ be the direct limit of Div $X$ along $\mu$; then $G_{\mu}$ is a totally ordered group (since $\mu$ is a maximal $\Delta$-filter), and the canonical homomorphism $L_{\mu}$ is lattice-preserving. Let $\nu_{\mu} \equiv L_{\mu} \circ \nu$. It was proved in [1] that $\nu_{\mu}$ is a valuation of $F$ whose valuation ring is $A_{M}$. If $\mu=x \in X$, then $G_{x}$ is, of course, the additive group of integers $Z$. Further, $G_{\mu}$ has been extensively studied [1]; thus the local ideal theory of $A$ is quite well known.

It will be convenient to let $\infty_{\mu} \equiv \nu_{\mu}(0)$ and to define $\infty_{\mu}>g$ for all $g \in G_{\mu}$. Let $\infty_{\mu}+g=\infty_{\mu}=\infty_{\mu}+g$ for all $g \in G_{\mu} \cup\{\infty\}$. Let $\operatorname{spec}\left(\operatorname{Div}^{+} X\right) \equiv\{\boldsymbol{Q}$ $\in J\left(\mathrm{Div}^{+} X\right): Q$ is prime $\}$. For $J \in J\left(\mathrm{Div}^{+} X\right)$ let $W(J) \equiv\{Q \in$ $\left.\operatorname{spec}\left(\operatorname{Div}^{+} X\right): J \subset Q\right\}$, and let $\left\{W(J): J \in J\left(\operatorname{Div}^{+} X\right)\right\}$ serve as the set of closed sets for the topology on $\operatorname{spec}\left(\operatorname{Div}^{+} X\right)$. Let $\operatorname{specm}\left(\operatorname{Div}^{+} X\right) \equiv\{J \in$ $J\left(\mathrm{Div}^{+} X\right): J$ is maximal $\} . \operatorname{specm}\left(\mathrm{Div}^{+} X\right)$ is a subset of $\operatorname{spec}\left(\mathrm{Div}^{+} X\right)$; let it be given the induced topology. For $J \in J\left(\mathrm{Div}^{+} X\right)$ let $V(J)=\left\{J^{\prime} \in\right.$ specm(Div $\left.\left.{ }^{+} X\right): J \subset J^{\prime}\right\} . J$ will be called local if $V(J)$ consists of a single point. From Banaschewski's Theorem (2.7) we have:

LEMMA 3.7. $\nu$ induces a homeomorphism of $\operatorname{spec} A(X)$ onto $\operatorname{spec}\left(\mathrm{Div}^{+} X\right)$. $I \in I(A)$ is local if and only if $\nu(I) \in J\left(\mathrm{Div}^{+} X\right)$ is local.

It is also clear that we can immediately obtain versions of (3.1)-(3.4) for $(\operatorname{Div} X) \cup\{\infty\}$. These results will be referred to as (3.1')-(3.4'), respectively.

4. Virtual-generators. Let $\tilde{G} \equiv \Pi_{\mu \in \delta x} G_{\mu}$ and let lattice and group operations on $\tilde{G}$ be defined pointwise; then $\tilde{G}$ is a lattice-ordered group. For $a \in \operatorname{Div} X$, let $\tilde{a}(\mu) \equiv L_{\mu}(a) \in G_{\mu}$, for each $\mu \in \delta X$; then $a \in \operatorname{Div} X \mapsto \tilde{a} \in$ $\tilde{G}$ is a lattice preserving group isomorphism into $\tilde{G}$. Let $\operatorname{Div} \delta X \equiv\{\tilde{a}$ : $a \in \operatorname{Div} X\}$. Let $\tilde{\infty} \equiv\left(\infty_{\mu}\right)_{\mu \in \delta X}$ be ordered so that $\tilde{\infty}>g$ for all $g \in \tilde{G}$, and let $\tilde{\infty}+g \equiv \tilde{g} \equiv g+\tilde{\infty}$, for all $g \in \tilde{G}$. Since $G_{\mu} \cup\left\{\infty_{\mu}\right\}$ is totally ordered, a dual ideal in it is just a nonempty subset $S$ for which $s \in S$ and $g \in G_{\mu}$, 
with $s<g$, implies $g \in S$. Let $H_{\mu} \equiv$ ddual ideals in $\left.G_{\mu} \cup\left\{\infty_{\mu}\right\}\right\} . H_{\mu}$ is, of course, totally ordered by inclusion. It will be convenient to order $H_{\mu}$ by anti-inclusion, i.e., for $S, S^{\prime} \in H_{\mu}$, define $S<S^{\prime}$ if and only if $S^{\prime} \subset S$. So ordered $g \in G_{\mu} \cup\left\{\infty_{\mu}\right\} \mapsto\left[g, \infty_{\mu}\right]\left(\equiv\left\{g^{\prime} \in G_{\mu} \cup\left\{\infty_{\mu}\right\}: g<g^{\prime}\right\}\right) \in H_{\mu}$ is an order-preserving injection into $H_{\mu}$, which will serve as an identification. Let $G_{\mu} \cup\left\{\infty_{\mu}\right\} \in H_{\mu}$ be denoted, for convenience, by $-\infty_{\mu}$. Clearly $H_{\mu}$ is closed under arbitrary nonempty unions and intersections; thus it is (Dedekind) complete and $G_{\mu}$ is dense in it. Given $S$ and $S^{\prime}$ in $H_{\mu}$, let $S+S^{\prime} \equiv\{s$ $+s^{\prime}: s \in S$ and $s^{\prime} \in S^{\prime}$; ; then $S+S^{\prime}$ is in $H_{\mu}$ and, as is easy to see, $H_{\mu}$ is a commutative semigroup under + , and that restricting the addition in $H_{\mu}$ to $G_{\mu}$ agrees with the group addition on $G_{\mu}$. Clearly $\infty_{\mu}+h_{\mu}=\infty_{\mu}$, for all $h_{\mu} \in H_{\mu} .-\infty_{\mu}+h_{\mu}=-\infty_{\mu}$, for all $h_{\mu}$ in $H_{\mu}$ less than $\infty_{\mu}$. Let $H \equiv$ $\Pi_{\mu \in \delta X} H_{\mu}$ and let order and addition on $H$ be defined pointwise; then $H$ is a complete lattice and an additive semigroup. Let $\tilde{\infty} \equiv\left(\infty_{\mu}\right)_{\mu \in \delta x}$ and let $-\infty \equiv(-\infty)_{\mu \in \delta X}$. The inclusion map of $\tilde{G}$ into $H$ preserves the lattice operations as well as + . For $k \in H$ let $J(k) \equiv\{a \in(\operatorname{Div} X) \cup\{\infty\}: \tilde{a}>$ $k\}$; then $J(k) \in \operatorname{di}(\operatorname{Div} X)$. Given $J \in \operatorname{di}(\operatorname{Div} X)$, let $L(J) \equiv \wedge_{a \in J} \tilde{a}$; then $L(J) \in H . k$ will be said to be approximable from above by divisors if $k=\bigwedge_{\tilde{a}>k} \tilde{a}$, where $\tilde{a}$ is understood to be in $\operatorname{Div} \delta X$. Let afa $(H) \equiv\{k \in H$ : $k$ approximable from above by divisors\}. Clearly Div $\delta X \subset$ afa $(H)$.

EXAMPLE 4.1. Let $D$ be infinite and in prop $\Delta$.

(1) Let $k \mid D \equiv 1$ ( 1 denoting the smallest positive element in $G_{\mu}$, for each $\mu \in \delta X$ ), and let $k \mid \delta X-D \equiv 0$. Clearly $k$ is in $H$. Let $h \mid \operatorname{cl}_{\delta X} D \equiv 1$ and $h \mid\left(\delta X-\mathrm{cl}_{\delta X} D\right) \equiv 0$; then $h \in H$ and $h=L(J(k))$, but $h \neq k$; thus $k$ is not in $\operatorname{afa}(H)$.

(2) Let $\mu \in \delta X-X$ and let $k^{\prime}(\mu) \equiv-1$, and let $k^{\prime} \mid(\delta X-\{\mu\}) \equiv 0$; then $L\left(J\left(k^{\prime}\right)\right)=0$, and so $k^{\prime} \notin \operatorname{afa}(H)$.

(3) Let $\mu$ be in $\delta X$, let $k^{\prime \prime}(\mu) \equiv 1$ and let $k^{\prime \prime} \mid(\delta X-\{\mu\}) \equiv 0$; then $k^{\prime \prime}$ is in $\operatorname{afa}(H)$. In fact $k^{\prime \prime}=L \nu\left(M_{\mu}\right)$.

Let $J \in \operatorname{di}(\operatorname{Div} X)$ and let $\mu \in \delta X$. Let $N_{\mu} \equiv \nu\left(M_{\mu}\right)$; then $N_{\mu} \in$ $\operatorname{specm}\left(\operatorname{Div}^{+} X\right)(2.7)$. Let $J_{\mu} \equiv J_{N_{\mu}}$ (See $\$ 2$ for the definition of $J_{N_{\mu}}$ ) Let $h \equiv L(J)$ and let $h_{\mu} \equiv L\left(J_{\mu}\right)$. Let $J_{\mu}$ (resp., $\left.h_{\mu}\right)$ be called $J$ (resp., $h$ ) localized at $\mu$.

LEMMA 4.1. Assume that $J \neq\{\infty\}$; then $h_{\mu}(\mu)=h(\mu)$, and for all $\mu^{\prime} \in \delta X$ $-\{\mu\}, h_{\mu}\left(\mu^{\prime}\right)=-\infty_{\mu^{\prime}}$.

Proof. Let $a \in J$ and let $b \in \operatorname{Div}^{+} X-N_{\mu} \cdot \tilde{b}(\mu)=0$, and hence $(a-$ $b) \sim(\mu)=\tilde{a}(\mu)$, showing that $h_{\mu}(\mu)=h(\mu)$. Since $J \neq\{\infty\}$ there exists $a \in J$ $-\{\infty\}$. Let $\mu^{\prime}$ be any point in $\delta X-\{\mu\}$. Let $g_{\mu^{\prime}}$ be an element in $G_{\mu^{\prime}}$. Let $\gamma_{\mu^{\prime}} \equiv \tilde{a}\left(\mu^{\prime}\right)-g_{\mu^{\prime}} \in G_{\mu^{\prime}}$. By Florack's Theorem, there exists $b \in\left(\operatorname{Div}^{+} X\right)-$ $N_{\mu}$ such that $\tilde{b}\left(\mu^{\prime}\right) \geqslant \gamma_{\mu^{\prime}}$; thus $(a-b)^{\sim}\left(\mu^{\prime}\right)<g_{\mu^{\prime}}$ and hence $h_{\mu}\left(\mu^{\prime}\right)=-\infty_{\mu^{\prime}}$, proving the lemma. 
Lemma 4.2. Let $J \in J(\operatorname{Div} X)$ and let $h \equiv L(J) \in H$; then $J=J(h)$.

Proof. Since $h \equiv \bigwedge_{a \in J} \tilde{a}, J \subset J(h)$. Since by assumption, $J$ is in $J(\operatorname{Div} X)$, there exists $d \in \operatorname{Div} X$ such that $d<a$, for all $a \in J$; thus $J \subset$ $\left(\mathrm{Div}^{+} X\right)+d$. By (3.2'),

$$
J=\bigcap_{\mu \in \delta X}\left(J_{\mu} \cap\left(\left(\operatorname{Div}^{+} X\right)+d\right)\right) .
$$

Let $a \in J(h)$; then, by definition, $\tilde{a}(\mu)>h(\mu)$, for all $\mu \in \delta X$. Since $J \subset$ $\left(\operatorname{Div}^{+} X\right)+d, h \geqslant \tilde{d}$, and as a consequence, $a \in\left(\operatorname{Div}^{+} X\right)+d$. Since $\tilde{a}(\mu)>$ $h(\mu)$, we may invoke (4.1) and know that $a \in J_{\mu}$ for all $\mu \in \delta X$. Using (*) we see that $a \in J$, proving that $J(h) \subset J$, and so proving the lemma.

We now come to one of the main theorems of the section.

Theorem 4.3. Let $J \in \operatorname{di}(\operatorname{Div} X)$, and let $h \equiv L(J) \in H$; then $J=J(h)$, proving that $h$ is in $\operatorname{afa}(H)$. Conversely, given $k \in \mathrm{afa}(H)$, then $L(J(k))=k$. Hence $L$ is an injection of $\operatorname{di}(\operatorname{Div} X)$ onto $\operatorname{afa}(H)$, whose inverse is $k \in \mathrm{afa}(H)$ $\mapsto J(k) \in \operatorname{di}(\operatorname{Div} X)$.

Proof. Clearly $J \subset J(h)$. Note that

$$
J=\bigcup_{d \in \operatorname{Div} x} J \cap J(\tilde{d}) .
$$

Clearly $J(\tilde{d})$ is in $J(\operatorname{Div} X)$.

$$
L(J \cap J(\tilde{d})) \equiv \bigwedge_{b \in J \cap J(\tilde{d})} \tilde{b}=\bigwedge_{a \in J}(\tilde{a} \vee \tilde{d})=\left(\bigwedge_{a \in J} \tilde{a}\right) \vee \tilde{d}=h \vee \tilde{d}
$$

By (4.2), $J \cap J(\tilde{d})=J(h \vee \tilde{d})$; thus (**) implies

$$
J=\bigcup_{d \in \operatorname{Div} X} J(h \vee \tilde{d})
$$

Let $a \in J(h)$. Clearly $a \in J(h \vee \tilde{a})$. Using (***), we see that $a \in J$, proving that $J(h) \subset J$, and hence that $J=J(h)$, and that $h \in \operatorname{afa}(H)$. Let $k \in$ $\operatorname{afa}(H)$; then $k$ is, by definition, $\bigwedge_{\tilde{a}>k} \tilde{a}$. Thus $L(J(k))=k$. The rest follows easily.

For $J \in \operatorname{di}(\operatorname{Div} X), L(J) \equiv h$ will be called the virtual generator of $J$, since $J=J(h)$. If $h=\tilde{a} \in \operatorname{Div} \delta X$ then $J=a+\operatorname{Div}^{+} X$, and $I \equiv \nu^{-1}(J)=f \cdot A$, where $\nu(f)=a$. In this case $h$ will be called a generator of $J$ and $J$ will be called principal. The first part of (4.3) thus states that every $J \in \operatorname{di}(\operatorname{Div} X)$ has a virtual-generator. We could thus go on to say that every $J \in \operatorname{di}(\operatorname{Div} X)$ is virtually principal. Let $\operatorname{afa}^{*}(H) \equiv\{h \in \mathrm{afa}(H)$ : there exists $d \in \operatorname{Div} X$ such that $\tilde{d}<h\}$, and let $\operatorname{afa}\left(H^{+}\right) \equiv\{h \in \operatorname{afa}(H): h>0\}$.

COROLLARY 4.4. $L$ is an order reversing injection of $\operatorname{di}(\operatorname{Div} X)$ onto $\operatorname{afa}(H)$ which takes $J(\operatorname{Div} X)$ onto $\mathrm{afa}^{*}(H)$, and $J\left(\operatorname{Div}^{+} X\right)$ onto afa $\left(H^{+}\right)$. 
Finally we have:

TheOREM 4.5. Let $I_{t} \in \operatorname{sam}(F)$, let $J_{t} \equiv \nu\left(I_{t}\right)$, and let $h_{t} \equiv L\left(J_{t}\right)$, for all $t \in T$, a set that contains 1 and 2. The following hold:

(0) $I_{1} \subset I_{2}, J_{1} \subset J_{2}$, and $h_{1}>h_{2}$ are equivalent;

(1) $\nu\left(\cap_{t \in T} I_{t}\right)=\bigcap_{t \in T} J_{t}$ and $L\left(\cap_{t \in T} J_{t}\right)=\bigvee_{t \in T} h_{t}$;

(2) $\nu\left(I_{1}+I_{2}\right)=J_{1} \wedge J_{2}$ and $L\left(J_{1} \wedge J_{2}\right)=h_{1} \wedge h_{2}$;

(3) $v\left(I_{1} \cdot I_{2}\right)=J_{1}+J_{2}$ and $L\left(J_{1}+J_{2}\right)=h_{1}+h_{2}$;

(4) $\nu\left(I_{1}: I_{2}\right)=J_{1}-J_{2}$ and $L\left(J_{1}-J_{2}\right)=\bigwedge_{\tilde{a}+h_{2}>h_{1}} \tilde{a}$;

(5) afa $(H)$, afa* $(H)$, and afa $\left(H^{+}\right)$are closed under arbitrary (nonempty) $\vee$, finite $\wedge$, and + ;

(6) $\nu(0)=\infty$ and $L(\{\infty\})=\tilde{\infty}$

(7) $\nu(A)=\left(\operatorname{Div}^{+} X\right) \cup\{\infty\}$ and $L\left(\left(\operatorname{Div}^{+} X\right) \cup\{\infty\}\right)=0$;

(8) $\nu(F)=(\operatorname{Div} X) \cup\{\infty\}$ and $L((\operatorname{Div} X) \cup\{\infty\})=-\tilde{\infty}$.

Proof. (0) was noted above. To check (1)-(4), note that the statements about $\nu$ were proved in $\$ 2$. To check the statements about $L$ it suffices to check them pointwise, where they are obvious. (1)-(4) imply (5). (6)-(8) are obvious.

$h \in \operatorname{afa}(H)$ we will be called principal if it is in (Div $\delta X) \cup\{\tilde{\infty}\}$. Now let $h \in \mathrm{afa}\left(H^{+}\right)$. Let $V(h) \equiv\{\mu \in \delta X: h(\mu)>0\}$. $h$ will be called local if $V(h)$ consists of a single point. $h$ will be called minimal if $h$ is local, with $V(h)=\{\mu\}$, and $h(\mu)=1$. $h$ will be called prime if it is local, with $V(h)=$ $\{\mu\}$, and if $\left\{g_{\mu} \in G_{\mu}:\left|g_{\mu}\right|<h(\mu)\right\}$ is a (necessarily convex) subgroup of $G_{\mu}$. (A definition of $h$ being primary can also be made using the criterion developed in [3].)

Theorem 4.6. Let $I \in I(A)$ and let $h \equiv L \nu(I) \in \mathrm{afa}(H)$. $I$ is local, maximal, prime (or primary) according as $h$ is local, minimal, prime (or primary).

Proof. The equivalence between $I$ local (resp., maximal) and $h$ local (resp., minimal) is obvious. The equivalence between $I$ prime and $h$ prime follows from results of Krull [17] and Henriksen [12]. (For a proof of the statements about primary objects see [3].)

5. Applications and further development. $I \in \operatorname{sam}(F)$ is called principal if there exists $f \in F$ such that $I=A f$.

THEOREM 5.1. Every finitely generated $I \in \operatorname{sam}(F)$ is principal, and thus of the form $A f \in I(F)$, for some $f \in F . I \neq 0$ if and only if $f \neq 0$. Assume that $I \neq 0$. $L \nu(I)=\nu(f)$; thus $L \nu(I) \in \operatorname{Div} \delta X . f$ is uniquely determined up to multiplication by $a$ unit in $A$. 
Proof. Clearly $\{0\}=A \cdot 0$. Assume $I \neq 0$, and let $i_{1}, \ldots, i_{n}$ be nonzero generators of $I$; then $I=A i_{1}+\ldots+A i_{n}$. Let $a_{j} \equiv \nu\left(i_{j}\right)$, for $1<j<n$, and let $a \equiv a_{1} \wedge \ldots \wedge a_{n} \in \operatorname{Div} X$. By 4.5(2), $h \equiv L \nu(I)=\tilde{a}_{1} \wedge \ldots \wedge \tilde{a}_{n}=\tilde{a} \in$ Div $\delta X$. By Florack's Theorem, there exists $f \in F^{*}$ such that $\nu(f)=a$. The rest is obvious.

$I \in I(F)$ is called invertible if there exists $I^{\prime} \in I(F)$ such that $I \cdot I^{\prime}=A$. The following are well known: if $I$ is invertible then $I^{\prime}=A: I$; the set of invertible ideals, inv $I(F)$, form a multiplication group; $\left\{A f: f \in F^{*}\right\}$ is a subgroup of inv $I(F)$; and invertible fractional ideals are finitely generated (see e.g. [19, p. 272]). Hence, using (5.1), we have:

TheOREM 5.2. inv $I(F)=\left\{A f: f \in F^{*}\right\}$; thus $L \nu$ is an isomorphism of inv $I(F)$ onto Div $\delta X$.

Theorem 5.3. Let $I \in \operatorname{sam}(F)$ be countably generated. There exists a set $\left(g_{n}\right)_{n \in N}$ of generators of $I$ such that $\nu\left(g_{n+1}\right)<\nu\left(g_{n}\right)$, for all $n \in N$. Hence $h \equiv L \nu(I)$ is $\wedge_{n \in N} \nu\left(g_{n}\right) \sim$. If $I \in I(A)$, then $g_{n+1} \mid g_{n}$ is in $A$, for all $n \in N$.

Proof. Let $\left(f_{n}\right)_{n \in N}$ be a set of generators of $I$ and let $b_{n} \equiv a_{1} \wedge \ldots \wedge a_{n}$. By (2.2), there exists $g_{n} \in I$ such that $\nu\left(g_{n}\right)=b_{n}$. Further, by (2.6), $\left(g_{n}\right)_{n \in N}$ generates $I$. Clearly $\nu\left(g_{n+1}\right)<\nu\left(g_{n}\right)$, for each $n \in N$. The rest is clear.

THEOREM 5.4. Let $I$ be a local ideal in $A$, with $V(I)=\{M\}$. Let $\mu \equiv Z(M)$. If $\mu \in X$ then $I$ is principal. If $\mu \in \delta X-X$, then I does not possess a countable set of generators. Finally, $Z(I)=\mu$, for all $\mu \in \delta X$.

Proof. By (4.6), $h$ is local. If $\mu \in X$ then $h(\mu) \in N$, and so $I$ is principal. Note that $Z(I)=\mu$ in this case. Now assume that $\mu \in \delta X-X$, and let $\tau \equiv Z(I)$. Since $h$ is local $\tau$ is contained in a unique element of $\delta X$; namely $\mu$. Let $D \in \tau \cap$ prop $\Delta . \tau \mid D$ is necessarily contained in a unique $\rho \in \beta D$; thus $\rho=\tau \mid D([8,10 \mathrm{H} 1])$. Hence $\tau=\mu$. Let $\left(f_{t}\right)_{t \in T}$ be a set of generators of $I$; then $\left(Z\left(f_{t}\right) \mid D\right)_{t \in T}$ is a filter basis of $\rho \in \beta D-D$. By $[8,4 \mathrm{G}], T$ is uncountable.

The following theorem has somewhat the same flavor. For $I \in I(A)$, let $V_{0}(I) \equiv V(I) \cap X$.

THEOREM 5.5. Let I be a nonzero ideal in $A$.

(1) $V_{0}(I)$ is either finite or countably infinite.

(2) If $V_{0}(I)$ is infinite then $V(I)$ contains a subspace that is homeomorphic to $\beta N$.

(3) If $V_{0}(f)$ is finite and $V(I)$ is infinite, then $V(I)-V_{0}(I)$ contains a subspace that is homeomorphic to $\beta N$.

(4) $V(I)$ is either finite or of power $2^{\mathrm{c}}, \mathrm{c}$ being the power of the continuum.

(5) The power of $\Delta$ is $\mathrm{c}$, and the power of $\delta X$ is $2^{\mathrm{c}}$. 
Proof. Since $I$ is nonzero there exists $f \in I-\{0\}$. Let $D \equiv Z(f)$; then $D \in$ prop $\Delta$. Using (1.2) it is clear that $D$ is either finite or countably infinite. Since $V_{0}(I) \subset D,(1)$ is proved. Since $V(I)$ is closed in $\delta X, \mathrm{cl}_{\delta X} V_{0}(I) \subset V(I)$. If $V_{0}(I)$ is infinite it is easy to see that $\mathrm{cl}_{\delta X} V_{0}(I)$ is homeomorphic to $\beta N$, proving (2). If $V_{0}(I)$ is finite and $V(I)$ is infinite, then $V(I)-V_{0}(I)$ is an infinite closed subset of $\operatorname{cl}_{\delta X} D$, a space homeomorphic to $\beta D$. By $[8,9.12]$, $V(I)-V_{0}(I)$ contains a copy of $\beta N$, proving (3). The power of $\beta N$ is $2^{\mathfrak{c}}$ [8, 9.3]. To see that the power of $\Delta$ is c, let $\left(U_{n}\right)_{n \in N}$ be as in (1.2). Given $D \in$ prop $\Delta, D \cap U_{n}$ is finite; thus the power of prop $\Delta$ is bounded above by $\mathrm{CN}_{0}{ }^{2}=\mathrm{c}$. Clearly it is bounded below by $\mathrm{c}$; thus $|\Delta|=c$. $\delta X=$ $\cup_{D \in \text { prop } \Delta} \operatorname{cl}_{\delta X} D$. For each $D \in$ prop $\Delta, \mathrm{cl}_{c X} D$ is either finite or by (1.5) and the above is of power $2^{c}$, according as $D$ is finite or infinite; thus $|\delta X|<2^{c}$. Since there exist infinite $D \in$ prop $\Delta, 2^{\mathrm{c}}=\left|\mathrm{cl}_{\delta X} D\right|<|\delta X|$, proving (5).

$I \in I(A)$ is fixed if and only if $V_{0}(I) \neq \varnothing$. Given a nonunit $f$ in $A$, then $A f$ is fixed. There follows an example of a nonprincipal fixed ideal in $A(C)$.

EXAMPLE 5.1. For all $w \in C$,

$$
\sin w=w \prod_{m=1}^{\infty}\left(1-w^{2} / m^{2} \pi^{2}\right) \equiv f(w) .
$$

For each $n \in N$ let

$$
f_{n}(w) \equiv w \prod_{m=1}^{n}\left(1-w^{2} / m^{2} \pi^{2}\right),
$$

and let $I$ be generated by $\left(f^{2} / f_{n}\right)_{n \in N} . V_{0}(I)=\{n \pi: n \in Z\} \equiv \pi Z$; thus $I$ is fixed. (supp $h$ ) $\cap \mathrm{C}$ is, of course, $\pi Z$ and for each $n \in Z, h(\pi n)=1$. However, for each $\mu \in\left(\mathrm{cl}_{\delta \mathrm{c}} \pi Z\right)-\pi Z, h(\mu)=2$; thus $f$ is not in $I$. Note further that $h \mid \delta \mathrm{C}-\mathrm{cl}_{\delta \mathrm{C}} \pi Z=0$.

Let $S$ be any nonempty subset of $\delta X$. Given $h, h^{\prime} \in \operatorname{afa}(H)$, we will write $h \sim_{s} h^{\prime}$ if $h\left|S=h^{\prime}\right| S$. Clearly this is an equivalence relation on $\operatorname{afa}(H)$. For $I$ and $I^{\prime}$ in $\operatorname{sam}(F)$ we will write $I \sim_{s} I^{\prime}$ if $L \nu(I) \sim_{s} L \nu\left(I^{\prime}\right)$. Thus $\sim_{s}$ is an equivalence relation on $\operatorname{sam}(F)$. Let $h_{t}$ and $h_{t}^{\prime}$ be in afa $(H)$, for all $t$ in some set $T$ containing 1 and 2 , such that $h_{t} \sim h_{t}^{\prime}$ for all $t \in T$. Then we have:

(1) $\bigvee_{t \in T^{h}} \sim_{s} \bigvee_{t \in T^{\prime}} h^{\prime}$,

(2) $h_{1} \wedge h_{2} \sim_{s} h_{1}^{\prime} \wedge h_{2}^{\prime}$, and

(3) $h_{1}+h_{2} \sim_{s} h_{1}^{\prime}+h_{2}^{\prime}$.

Using (4.5), this translates into statements about $\sim_{s}$ on $\operatorname{sam}(F)$. One of the most interesting choices for $S$ is $X$ itself. This case was considered by Schilling [18] in 1946 in case $X=C$. If $S=X$, let the subscript $S$ be suppressed. Following Schilling, $I$ and $I^{\prime}$ in $\operatorname{sam}(F)$ will be called quasi-equal if $I \sim I^{\prime}$. Let $I \sim I^{\prime}$. Clearly if $I$ is in $I(F)$ (or $I(A)$ ) then so also is $I^{\prime}$. Clearly $I \in I(A)$ is a free ideal in $A$ if and only if $I \sim A$. This sets the stage for the main decomposition theorem. 
THeOREM 5.6. Let $I \in I(F)-\{0\}$ and let $h \equiv L \nu(I)$.

(1) There exists a unique $b \in \operatorname{Div} X$ such that $h \mid X=b$.

(2) $h>\tilde{b}$.

(3) $J(\equiv \nu(I)) \subset J(\tilde{b})$. Let $f \in F^{*}$, such that $\nu(f)=b$; then $A f=\nu^{-1}(J(\tilde{b}))$.

(4) $I \subset$ Af and $I \sim A f$.

(5) $I: A f \equiv I^{\prime}$ is a free ideal in $A$.

(6) $A f \cdot I^{\prime}=I$.

(7) Given any free ideal $I^{\prime \prime}$ in $I(A)$ and any $g \in F^{*}$, then $A g \cdot I^{\prime \prime} \subset A g$, and Ag $\cdot I^{\prime \prime} \sim A g$.

(8) Af is the largest element in $I(F)$ that is quasi-equal to $I$.

Proof. Since $I$ is in $I(F)-\{0\}$ there exists $d \in \operatorname{Div} X$ such that $h+\tilde{d}>$ 0 (by the definition of a fractional ideal). Let $t \in F^{*}$ such that $\nu(t)=d$, and let $I_{1} \equiv I \cdot(A t) \in I(F)$. Since $h+\tilde{d}>0, I_{1}$ is in $I(A)$. Let $h_{1} \equiv L \nu\left(I_{1}\right)$; then, by $4.5(3), \quad h_{1}=h+\tilde{d}$. $\operatorname{supp}\left(h_{1}\right)=V\left(I_{1}\right) ;$ thus $\left(\operatorname{supp}\left(h_{1}\right)\right) \cap X \equiv D \in \Delta$. Since $t \neq 0$, and since $I \neq\{0\}, I_{1} \neq\{0\}$, and so $D \in$ prop $\Delta$. As a result, $h_{1} \mid X \equiv d_{1}$ is in $\operatorname{Div}^{+} X$. Let $J_{1} \equiv \nu\left(I_{1}\right)$. Clearly $a \in J_{1}$ implies $a>d_{1}$; thus $h_{1}$, which equals $\bigwedge_{a \in J_{1}} \tilde{a}$, is greater than or equal to $\tilde{d}_{1}$. Since $d \in \operatorname{Div} X$, $h=h_{1}+(-d)$. Let $b \equiv d_{1}-d$, and note that $b$ is in Div $X$, that $h \mid X=b$ and, since $h_{1} \geqslant \tilde{d}_{1}$, that $h \geqslant \tilde{b}$, proving (1) and (2). Clearly (1) and (2) imply (3) and (4). As to (5), let $h^{\prime} \equiv L \nu\left(I^{\prime}\right)$. By 4.5(4), $h^{\prime}=\bigwedge_{\tilde{a}+\tilde{b}>h} \tilde{a}$, which equals $\bigwedge_{\tilde{a}>\tilde{h}+(-b)^{-}} \tilde{a}$. Since $\left(h+(-b)^{\sim}\right)\left|X=0, h^{\prime}\right| X=0$, and so $I^{\prime}$ is a free ideal in $A$. To prove (6), using 4.5(3) and 4.5(4), we see that

$$
L \nu\left(A f \cdot I^{\prime}\right)=b+\bigwedge_{\tilde{a}+\tilde{b}>h} \tilde{a}=\bigwedge_{\tilde{a}+\tilde{b}>h} \tilde{a}+\tilde{b}=h .
$$

By (4.3), $A f \cdot I^{\prime}=I$, proving (6). To prove (7), let $h^{\prime \prime} \equiv L \nu\left(I^{\prime \prime}\right)$. Since $I^{\prime \prime}$ is free, $h^{\prime \prime} \mid X=0$. By $4.5(3)$,

thus $A g \cdot I^{\prime \prime} \subset A g$. Since

$$
\operatorname{L\nu }\left(A g \cdot I^{\prime \prime}\right)=\operatorname{L\nu }(g)+h^{\prime \prime}>\operatorname{L\nu }(g)
$$

$$
\operatorname{L\nu }\left(A g \cdot I^{\prime \prime}\right)|X=L \nu(g)| X,
$$

$A g \cdot I^{\prime \prime} \sim A g$, proving (7). To prove (8) note that $I^{\prime} \subset A$ and that $A$ is free.

Let $I \in I(F)-\{0\}$. By (5.6), $\theta(I) \equiv L \nu(I) \mid X$ is in $\operatorname{Div} X$.

CoROLlaRY 5.7. Let $I_{1}$ and $I_{2}$ be in $I(F)-\{0\}$.

(1) $\theta$ maps $I(F)-\{0\}$ onto $\operatorname{Div} X$ and $I(A)-\{0\}$ onto $\mathrm{Div}^{+} X$;

(2) $\theta\left(I_{1}\right)=\theta\left(I_{2}\right)$ if and only if $I_{1} \sim I_{2}$;

(3) $I_{1} \subset I_{2}$ implies $\theta\left(I_{1}\right) \geqslant \theta\left(I_{2}\right)$;

(4) $\theta\left(I_{1} \cap I_{2}\right)=\theta\left(I_{1}\right) \vee \theta\left(I_{2}\right)$;

(5) $\theta\left(I_{1}+I_{2}\right)=\theta\left(I_{1}\right) \wedge \theta\left(I_{2}\right)$;

(6) $\theta\left(I_{1} \cdot I_{2}\right)=\theta\left(I_{1}\right)+\theta\left(I_{2}\right)$

(7) $\theta\left(I_{1}: I_{2}\right)=\theta\left(I_{1}\right)-\theta\left(I_{2}\right)$;

(8) $\theta^{-1}(0)=\{I \in I(A)$ : $I$ is free $\}$. 
This follows easily from (4.5) and (5.6).

EXAMPLE 5.2. (1) Helmer considered the following example in 1940 [10] for $X=$ C. For each $n \in N$, let $g_{n}(w) \equiv \sin \left(w / 2^{n-1}\right)$, for all $w \in$ C. Let $I$ be generated by $\left(g_{n}\right)_{n \in N} . I$ is a proper ideal in $A$. The zero set of $g_{n}$ is $2^{n-1} \pi Z$. Let $b_{n} \equiv \nu\left(g_{n}\right)$, for each $n \in N$ and note that $\wedge_{n \in N} b_{n} \equiv b$ is in $\mathrm{Div}^{+} \mathrm{C}$, that it is nonzero only at $0 \in \mathbf{C}$, and that $b(0)=1$. Let $g(w) \equiv w$, for all $w \in \mathbf{C}$; then $b=\nu(g)$. Clearly $I \subset(g)$ and $I \sim(g)$. Helmer noted that $I \neq(g)$. By (5.6), $I=A g \cdot I^{\prime}$, where $I^{\prime}$ is a free ideal in $A(C)$. Let $h^{\prime} \equiv L \nu\left(I^{\prime}\right)$. To describe $h^{\prime}$ completely let $\delta \equiv\left\{D \in \Delta\right.$ : there exists $n \in N$ such that $\left.2^{n-1} \pi Z \subset D\right\}$; then $\delta$ is a $\Delta$-filter. Let $T \equiv\{\mu \in \delta C: \delta \subset \mu\}$. Then $h^{\prime} \mid T=1$ and $h^{\prime} \mid \delta C-T$ $=0$.

(2) Returning to Example 5.1, by (5.6) $I=A f \cdot I^{\prime \prime}$, where $f(w) \equiv \sin w$ for all $w \in \mathrm{C}$, and $I^{\prime \prime}$ is a free ideal in $A(C)$. Let $h^{\prime \prime} \equiv L \nu\left(I^{\prime \prime}\right) . h^{\prime \prime}$ is supported on $\left(\mathrm{cl}_{\delta \mathrm{C}} \pi Z\right)-\pi Z$, a space homeomorphic to $\beta N-N$, where its value is 1 . Thus $I \sim A f$ and $I \varsubsetneqq(f)$.

(5.6) and (5.7) focus our attention on fr $I(A)$, the set of free ideals of $A$. Using (4.5), we can easily see that $\operatorname{fr} I(A)$ is closed under nonempty intersection, + , and $\cdot$ Let $I_{j} \in$ fr $I(A)$, let $h_{j} \equiv L \nu\left(I_{j}\right)$, let $I \equiv I_{1}: I_{2}$, and let $h \equiv L \nu(I)$.

THEOREM 5.8. $I$ is a free ideal in $A$.

Proof. By 4.5(4), $h=\bigwedge_{\tilde{a}+h_{2}>h_{1}} \tilde{a}$. Let $a \in \operatorname{Div} X$ such that

$$
\tilde{a}+h_{2} \geqslant h_{1} \text {. }
$$

Restricting (*) to $X$ gives us $a+0>0$ : i.e., $a>0$; thus $I \in I(A)$. Let $x \in X$ and let $b$ be the divisor such that $b(x)=a(x)$ and $b \mid X-\{x\}=0 . c \equiv a-b$ $\in \operatorname{Div}^{+} X$, then $\tilde{c}+h_{2}>h_{1}$ and $c(x)=0$; thus $h(x)=0$, proving that $I$ is free.

The following lemma must surely be well known. (Cf., e.g., [19, p. 147].)

LEMMA 5.9. Let $\left(I_{t}\right)_{t \in T}$ be a nonempty family in $\operatorname{sam}(F)$ and let $I_{2} \in$ $\operatorname{sam}(F)$; then $\left(\cap_{t \in T} I_{t}\right): I_{2}=\cap_{t \in T}\left(I_{t}: I_{2}\right)$.

Proof. $f \in\left(\cap_{t \in T} I_{t}\right): I_{2}$ if and only if $f I_{2} \subset I_{t}$, for all $t \in T$, proving the lemma.

LEMMA 5.10. If $I_{1}$ is local with $h_{1}(\mu)>0$; then $h\left(\mu^{\prime}\right)=0$ for all $\mu^{\prime} \in \delta X-$ $\{\mu\}$ (where $I_{1}, h_{1}$, and $h$ are defined above (5.8)). Hence $I$ is either local or is $A$.

Proof. Let $\mu^{\prime} \in \delta X-\{\mu\}$. There exist $D \in \mu$ and $D^{\prime} \in \mu^{\prime}$ such that $D \cap D^{\prime}=\varnothing$. There exists $a \in \operatorname{Div}^{+} X$ such that $a \mid D^{\prime}=0$ and $\tilde{a}(\mu)+h_{2}(\mu)$ $>h_{1}(\mu)$; thus $\tilde{a}+h_{2}>h_{1} .0=\tilde{a}\left(\mu^{\prime}\right)>h\left(\mu^{\prime}\right)$. Sine $I \subset A$ (by (5.8)), $h\left(\mu^{\prime}\right)>$ 0 , proving that $h\left(\mu^{\prime}\right)=0$. 
THEOREM 5.11. $I_{1}: I_{2}=\cap_{M \in V\left(I_{1}\right)}\left(\left(\left(I_{1}\right)_{M} \cap A\right): I_{2}\right)$, where $\left(I_{1}\right)_{M} \cap A$ is either local and free, or is $A$.

Proof. By (3.4), $I_{1}=\cap_{M \in V\left(I_{1}\right)}\left(\left(I_{1}\right)_{M} \cap A\right)$, each $\left(I_{1}\right)_{M} \cap A$ being local and necessarily free. By (5.9), $I_{1}: I_{2}=\cap_{M \in V\left(I_{1}\right)}\left(\left(\left(I_{1}\right)_{M} \cap A\right): I_{2}\right)$. By (5.10), $\left(\left(I_{1}\right)_{M} \cap A\right): I_{2}$ is local and free or is $A$.

Let $W \equiv \delta X-X$. Let us now consider the equivalence relation $\sim_{W}$ on afa $(H)$, on $\operatorname{sam}(F)$, on $I(F)$, and on $I(A)$.

THEOREM 5.12. (1) Given $f \in F^{*}$, with $b \equiv \nu(f)$ having finite support, then $A f \sim_{W} A$. Let $I \in I(A)-\{0\}$.

(2) If $I \sim_{W} A$, then there exists $f \in A^{*}$, whose support is finite, such that $I=A f$.

(3) For any $I \in I(A)-\{0\}$, there exists a unique free ideal $I^{\prime \prime}$ in $A$ such that $I \sim_{W} I^{\prime \prime}$.

Proof. (1) Let $D_{0} \equiv \operatorname{supp}(b)$. By hypothesis, $D_{0}$ is a finite subset of $X$; thus $\operatorname{cl}_{\delta X} D_{0}=D_{0}$, and so $\tilde{b} \mid W=0$. Hence $A f \sim_{W} A$, proving (1). Given $I \in I(A)$ - $\{0\}$, we may apply (5.6) and know that there exists $f \in A^{*}$ and $I^{\prime} \in$ fr $I(A)$ such that $I=A f \cdot I^{\prime}$, with $b=X \mid h \in \operatorname{Div} X$, where $b \equiv \nu(f), h \equiv$ $L \nu(I), h^{\prime} \equiv L \nu\left(I^{\prime}\right), h=\tilde{b}+h^{\prime}$, and $h^{\prime} \mid X=0$.

(2) Since, by hypothesis, $I \sim_{W} A, h \mid W=0$; thus $h^{\prime}|W=(-b) \sim| W<0$. Hence $h^{\prime} \mid W=0$. Since $h^{\prime} \mid X=0, h^{\prime}=0$, and as a result $I=A f$. Were $\operatorname{supp}(b)$ infinite there would exist $\mu \in W$ such that $\tilde{b}(\mu)>0$, and hence $h(\mu)$ $(=\tilde{b}(\mu))>0$, which is absurd. Thus $\operatorname{supp}(b)$ is finite.

(3) Let $\left(U_{n}\right)_{n \in N}$ be as described in (1.2). For $a \in \operatorname{Div}^{+} X$ and $n \in N$, let $a_{n}$ equal $a$ on $X-U_{n}$, and let $a_{n} \mid U_{n}=0 . a_{n} \in \operatorname{Div}^{+} X$, and $0<a_{n+1}<a_{n}<a$, for all $n \in N$. Let $k \equiv \bigwedge_{n \in N, a \in J(h)} \tilde{a}_{n}$; then, by definition, $k \in \operatorname{afa}(H)$. Clearly $k|W=h| W$ and $k \mid X=0$. Let $I^{\prime \prime} \equiv \nu^{-1}(J(k))$; then $I^{\prime \prime} \in \operatorname{fr} I(A)$ and $I \sim_{W} I^{\prime \prime}$. Clearly $I^{\prime \prime}$ is uniquely determined by $I$.

$A(X)$ has, of course, a natural topology on it, namely the topology of uniform convergence of compacta, under which it is complete. Schilling [18] showed that every closed ideal in $A(C)$ is principal. Adapting his argument to $X$ and, as always, using Florack's Theorem we obtain:

Schinling's Lemana 5.13. Let $I$ be a free ideal in $A$, then its closure $\bar{I}$ in $A$ is $A$ itself.

Proof. Since $I$ is free, $I \neq\{0\}$. Let $g \in I-\{0\}$, and let $D \equiv Z(g)$. If $D=\varnothing$, then $g$ is a unit in $A$ and so $I=A$, and hence $I=I=A$. Assume that $D \neq \varnothing$ and, at first, that $D$ is finite. Since $I$ is free, there exists $f \in I$ such that $D \cap Z(f)=\varnothing$. By Helmer's Lemma $(2.5),(f, g)=1$, and so $I=A=\bar{I}$. Assume now that $D$ is infinite. Since $g \neq 0, D \in$ prop $\Delta$; thus $D$ is countable. Let $n \in N \mapsto x_{n} \in D$ be a bijection. Since $I$ is free there exists 
$f_{n} \in I$ for which $f_{n}\left(x_{n}\right) \neq 0$, for each $n \in N$. By Florack's Theorem, there exists $f \in A$ for which $Z(f)=D$ and $\nu(f) \mid D=1$. Let $\left(U_{n}\right)_{n \in N}$ be as described in (1.2). By Florack's Theorem there exists $k_{n} \in A$ for which $Z\left(k_{n}\right)=$ $\left\{x_{n}\right\}$ and $\nu\left(k_{n}\right)\left(x_{n}\right)=1$, for each $n \in N$. Since $f_{n} \in I$ and $f / k_{n} \in A$; thus $h_{n} \equiv f_{n} f / k_{n}$ is in $I$. Since $\bar{U}_{n}$ is compact, $h_{n}$ is bounded on this set; thus there exists $c_{n} \in \mathbf{C}-\{0\}$ such that $\sup \left\{\left|c_{n} h_{n}(x)\right|: x \in \bar{U}_{n}\right\}<2^{-n}$, for each $n \in N$. Now let $x \in \bar{U}_{m}$. For $n>m, x \in \bar{U}_{n}$; thus $\left|c_{n} h_{n}(x)\right|<2^{-n}$. Hence $\sum_{n=1}^{\infty} c_{n} h_{n}$ converges on $X$ to some $h \in A$. By construction, $h \in \bar{I}$. It is easily seen that $\bar{I}$ is an ideal in $A . c_{n} h_{n}\left(x_{j}\right)=0$ if and only if $n \neq j$; thus $h\left(x_{n}\right) \neq 0$, for each $n \in N$, and so $Z(h) \cap D=\varnothing$. By Helmer's Lemma, $(g, h)=1$; thus $\bar{I}=A$, proving the lemma.

SCHILlING'S TheOREM 5.14. Let I be a closed ideal in $A$; then $I$ is principal.

Proof. If $I=A$ or 0 , then it is principal. Assume that $I$ is proper and nonzero. By (5.6), $I=A f \cdot I^{\prime}$, where $f \in A-\{0\}$, and $I^{\prime}$ is a free ideal in $A$. By (5.6(5)), $I^{\prime}=I: A f\left(=I \cdot f^{-1}\right)$. To show that $I^{\prime}$ is closed in $A$, let $g_{n} \in I^{\prime}$, for all $n \in N$, and let $\left(g_{n}\right)_{n \in N}$ converge to some $g \in A$. Let $h_{n}=f g_{n}$, for all $n \in N$, and let $h=f g$; then $h_{n} \in I$, for all $n \in N$; and $h \in A$. Let $K$ be a compact subset of $X$. Let $B \equiv \sup _{x \in K}|f(x)|$. For all $x \in K$,

$$
\left|h_{n}(x)-h(x)\right|<B\left|g_{n}(x)-g(x)\right| \text {. }
$$

Since $\left(g_{n}\right)_{n \in N}$ converges to $g$ on $X,\left(h_{n}\right)_{n \in N}$ converges to $h$ on $X$. Since $I$ is closed, $h \in I$, and so $g=h / f \in I^{\prime}$, proving that $I^{\prime}$ is closed. By Schilling's Lemma (5.13), we know that $I^{\prime}=A$ and hence $I=A f \cdot A=A f$, proving the theorem.

Corollary 5.15. Let $I \in I(A)-\{0\}$. The following are equivalent: $I$ is principal, $I$ is invertible in $I(F), I$ is closed in $A$.

In his dissertation, Kelleher [15] considered subrings $B$ of $F$ that contain $A$, calling such rings $A$-rings. Clearly the set of $A$-rings, $\operatorname{aring}(F)$, when partially ordered under inclusion, is inductive, closed under intersection, and has $A$ as its least element and $F$ as its greatest. Given a multiplication system $S$ of $A-\{0\}$, that contains 1 , then $S^{-1} A$ is an $A$-ring. Thus, given $P \in \operatorname{spec} A$, $A_{P}$ is an $A$-ring. Let $B \in \operatorname{sam}(F)$. Clearly $B$ is an $A$-ring if and only if (i) $1 \in B$, and (ii) $B$ is closed under multiplication. Let $J \equiv \nu(B)$; then $B$ is an $A$-ring if and only if (i') $0 \in J$, and (ii') $a, b \in J$ and $a, b<0$ implies $a+b \in J$. Let $h \equiv L(J) . B$ is an $A$-ring if and only if (i") $h<0$, and (ii") $h<\tilde{a}, \tilde{b}<0$ implies $h<\tilde{a}+\tilde{b}$. Assume now that $B$ is an $A$-ring. Let $\Gamma(B)$ (or $\Gamma$ for short) be defined to be $\left\{a \in \operatorname{Div} X: h<\tilde{a} \wedge(-a)^{\sim}\right\}$. For $a \in$ Div $X$ let $|a| \equiv a \vee(-a)$. Clearly $|a|>0,|a|=0$ implies $a=0,|a+b|<$ $|a|+|b|$, and $a \wedge(-a)=-|a|$; for all $a, b \in \operatorname{Div} X$. A subgroup $G$ of Div $X$ is called absolutely convex if $g \in G$ and $d \in \operatorname{Div} X$ such that $|d|<|g|$ 
implies $d \in G$. Let $U(B)$ denote the group of units of $B$. The following is easily seen.

LEMMA 5.16. $\Gamma$ is an absolutely convex subgroup of $\operatorname{Div} X$, and $\nu(U(B))=\Gamma$.

The following was proved by Kelleher [15, (3.2.6)] by a different argument. Let $S \equiv U(B) \cap A$.

TheOREM 5.17. $B=S^{-1} A$.

Proof. Clearly $S^{-1} A \subset B$. Let $f \in B-\{0\}$, and let $\nu(f)=a$. As usual let $a^{+} \equiv a \vee 0$ and $a^{-} \equiv a \wedge 0$; then $a=a^{+}+a^{-}$. Since $a^{+}>0$ there exists $r \in A$ for which $\nu(r)=a^{+}$. Since $f \in B, h<\left(a^{-}\right)^{-}<0$; thus there exists $s \in S$ for which $\nu(s)=-a^{-}$. Hence $\nu(r / s)=a^{+}+a^{-}=a=\nu(f)$. There must then exist a unit $u$ in $A$ such that $f=u r / s$. Since $A \subset B, u r \in B$, and we conclude that $B \subset S^{-1} A$, proving the theorem.

For $\mu \in \delta X$ let $\Gamma_{\mu} \equiv L_{\mu}(\Gamma)$. By (5.16), $\Gamma_{\mu}$ is a convex (= isolated) subgroup of $G_{\mu}$. Clearly $h(\mu)=0$ if and only if $\Gamma_{\mu}=0, h(\mu)=-\infty_{\mu}$ if and only if $\Gamma_{\mu}=G_{\mu}$; and hence $-\infty_{\mu}<h(\mu)<0$ if and only if $0 \varsubsetneqq \Gamma_{\mu} \varsubsetneqq G_{\mu}$. If $\mu \in X$ then $G_{\mu}$ is isomorphic to $Z$ and hence either $h(\mu)=0$ or $h(\mu)=-\infty_{\mu}$. Let $Q_{\mu} \equiv\left\{f \in F: \nu_{\mu}(f)>\gamma_{\mu}\right.$ for all $\left.\gamma_{\mu} \in \Gamma_{\mu}\right\}$. Krull showed, in his classic paper on valuation theory [17], that $Q_{\mu}$ is a prime ideal in the valuation ring $A_{M}$, where $M=Z^{-1}(\mu)$. Let $P_{\mu} \equiv Q_{\mu} \cap A$; then of course $P_{\mu}$ is in $\operatorname{spec} A$. Clearly the following holds. $h(\mu)=0$ if and only if $P_{\mu}=M_{\mu} \cdot h(\mu)=-\infty_{\mu}$ if and only if $P_{\mu}=\{0\}$. Thus $0 \varsubsetneqq P_{\mu} \varsubsetneqq M_{\mu}$ if and only if $-\infty \mu_{\mu}<h(\mu)<0$. Clearly if $\mu \in X, P_{\mu}$ is either 0 or $M_{\mu}$. An $S$-ideal in $A$ is an ideal of $A$ that does not meet $S$. Let $\delta_{B} X \equiv\left\{\mu \in X: h(\mu)>-\infty_{\mu}\right\}$. If $B=F, \delta_{B} X=\varnothing$. Assume henceforth that $B \neq F$; then $\delta_{B} X \neq \varnothing$.

LEMMA 5.18. $\left\{P_{\mu}: \mu \in \delta_{B} X\right\}$ is the set of all maximal $S$-ideals of $A$.

Proof. Let $\mu \in \delta_{B} A$. One sees from the definition of $P_{\mu}$ that it is the largest local ideal of $A$ that does not meet $S$, and that is contained in $M_{\mu}$. Since every ideal of $A$ is the intersection of local ideals (3.4), $P_{\mu}$ is indeed a maximal $S$-ideal. Conversely, let $I$ be a maximal $S$-ideal in $A$. By (3.1), $I=$ $\cap_{M \in V(I)}\left(I_{M} \cap A\right)$. Let $M_{\mu} \in V(I)$. Since $I \cap S=\varnothing, I \subset P_{\mu}$; thus $P_{\mu} \neq 0$, and so $h(\mu)>-\infty_{\mu}$ and hence $\mu \in \delta_{B} X$. Since $P_{\mu}$ is an $S$-ideal and $I$ is a maximal $S$-ideal, $I=P_{\mu}$, proving the lemma.

The following was also proved by Kelleher; again our proofs differ.

TheOREM 5.19. $B=\bigcap_{\mu \in \delta_{B} X} A_{P_{\mu}}$.

Proof. Let $f \in B$. By (5.17), $B=S^{-1} A$; thus there exist $r \in A$ and $s \in S$ such that $f=r / s$. Since $s \in S$ we see, using (5.18), that $f \in A_{P_{\mu}}$, for all $\mu \in \delta_{B} X$. Conversely let $f \in A_{P_{\mu}}$, for all $\mu \in \delta_{B} X$. By (2.1), there exists $a$, $b \in A$, with $b \neq 0$ and $(a, b)=1$, such that $f=a / b$. For all $\mu \in \delta_{B} X$, 
$b \notin P_{\mu}$ so $\nu\left(b^{-1}\right)^{\sim} \geqslant h$, and so $b \in S$, proving that $f \in S^{-1} A$, which by (5.17), is $B$, proving the theorem.

Let $\tilde{\Gamma} \equiv\{\tilde{a}: a \in \Gamma\}$. $\tilde{\Gamma}$ is a subgroup of $\operatorname{Div} \delta X$. For $\tilde{a} \in \operatorname{Div} \delta X$ let $|\tilde{a}| \equiv|a|^{\sim}$; then given $\tilde{a} \in \tilde{\Gamma}$, and $\tilde{b} \in \operatorname{Div} \delta X$ such that $|\tilde{b}|<|\tilde{a}|$ then $\tilde{b} \in \tilde{\Gamma}$; i.e., $\tilde{\Gamma}$ is an absolutely convex subgroup of Div $\delta X$. Clearly $\tilde{\Gamma}=\{\tilde{a} \in \operatorname{Div} \delta X$ : $h<-|\tilde{a}|\}$. Let $\varphi$ be the canonical homomorphism of Div $\delta X$ onto $(\operatorname{Div} \delta X) / \tilde{\Gamma} \equiv \Omega$. The order on Div $\delta X$ induces an order on $\Omega$, under which it is a lattice-ordered group. Let $\tau \equiv \varphi \circ \nu$; then $\tau$ is a homomorphism of $F^{*}$ onto $\Omega$, having $U(B)$ as kernel and such that $\tau^{-1}\left(\Omega^{+}\right)=B-\{0\}$, where $\Omega^{+} \equiv\{\omega \in \Omega: \omega \geqslant 0\}$. Let $\hat{\infty}$ be an element greater than all $\omega \in \Omega$, and let $\hat{\infty}+\omega=\hat{\infty}=\omega+\hat{\infty}$, for all $\omega \in \Omega \cup\{\hat{\infty}\}$. Let $\tau(0) \equiv \hat{\infty}$; then $\tau(f g)=$ $\tau(f)+\tau(g)$ and $\tau(f \pm g)>\tau(f) \wedge \tau(g)$ (by (2.4)). Div $\delta X$ is a subgroup of $\tilde{G} \equiv \Pi_{\mu \in \delta X} G_{\mu} . \tilde{\Gamma}$ is also a subgroup of $\tilde{G}$. $\Omega$ may be considered to be a subgroup of $\Pi_{\mu \in \delta X} G_{\mu} / \Gamma_{\mu}$. Since $\mu \in \delta X-\delta_{B} X$ implies $G_{\mu} / \Gamma_{\mu}=0$ it is more convenient to think of $\Omega$ as a subgroup of $\Pi_{\mu \in \delta_{B} X} G_{\mu} / \Gamma_{\mu}$. For each $\mu \in \delta_{B} X$ let $\rho_{\mu}$ be the projection of $\Omega$ onto $G_{\mu} / \Gamma_{\mu}$; then $\rho_{\mu}$ is an order-preserving homomorphism. Let $\tau_{\mu} \equiv \rho_{\mu} \circ \tau\left(=\rho_{\mu} \circ \varphi \circ \nu\right)$. Let $R_{\mu} \equiv B \cap Q_{\mu}$; then $R_{\mu} \in$ $\operatorname{spec} B$, and $R_{\mu} \cap A=P_{\mu}$. Let $\hat{\infty}_{\mu}$ be an element greater than all $t \in G_{\mu} / \Gamma_{\mu}$ $\equiv \Omega_{\mu}$; and let $\hat{\infty}_{\mu}+t \equiv \hat{\infty}_{\mu} \equiv t+\hat{\infty}_{\mu}$, for all $t \in \Omega_{\mu} \cup\left\{\hat{\infty}_{\mu}\right\}$. Let $\tau_{\mu}(0) \equiv$ $\hat{\infty}_{\mu}$.

THEOREM 5.20. For each $\mu \in \delta_{B} X, \tau_{\mu}$ is a valuation of $F$ whose valuation ring is $B_{R_{\mu}}$ and whose value group is $\Omega_{\mu}$. $R_{\mu}$ is thus a maximal ideal of $B$.

Proof. By definition, $\tau_{\mu}$ is a homomorphism of $F^{*}$ onto $\Omega_{\mu}$. Using (2.4) and the fact that $\varphi$ and $\rho_{\mu}$ are order preserving, one easily sees that $\tau_{\mu}$ is a valuation. (See e.g. [20, p. 43] for details.) Let $C_{\mu} \equiv\left\{f \in F: \tau_{\mu}(f)>0\right\}$; then, of course, $C_{\mu}$ is the valuation ring of $\tau_{\mu}$. Let $f \in B_{R_{x}}$. By definition, there exist $a \in B$ and $b \in B-R_{\mu}$ such that $f=a / b$. Since $a \in B, L \nu(a)>h$ and so $L_{\mu}(\nu(a))>h(\mu)$; thus $\tau_{\mu}(a)>0$ and so we see that $B \subset C_{\mu}$. Since $b \in B-$ $R_{\mu}, \tau_{\mu}(b)=0$. Hence $\tau_{\mu}(f)>0$, and so $B_{R_{\mu}} \subset C_{\mu}$. Conversely let $f \in C_{\mu}$. By definition $\tau_{\mu}(f) \geqslant 0$. By the definition of order on $\Omega_{\mu}\left(\equiv G_{\mu} / \Gamma_{\mu}\right)$ there exists $\gamma_{\mu} \in \Gamma_{\mu}$ such that $\nu_{\mu}(f)+\gamma_{\mu} \geqslant 0$. By definition of $\Gamma_{\mu}$ there exists $\gamma \in \Gamma$ so that $L_{\mu}(\gamma)=\gamma_{\mu}$. By (5.16), there exists $u \in U(B)$ so that $\nu(u)=\gamma$. As a consequence, $\nu_{\mu}(u f)>0$. By (2.1) there exist $a, b \in A$ such that $u f=a / b$ and $(a, b)=1$. Since $\nu_{\mu}(u f)>0, \nu_{\mu}(a)>\nu_{\mu}(b)$. Were $\nu_{\mu}(b)>0$, then $\nu_{\mu}(a)$ would be positive and so both $a$ and $b$ would be in $M_{\mu}$, implying that $1 \in M_{\mu}$, which is absurd. Hence $\nu_{\mu}(b)=0$, proving that $b \in A-P_{\mu} \subset B-R_{\mu}$. As a consequence $f \in B_{R_{\mu}}$, and so we see that $C_{\mu}=B_{R_{x}}$. To see that $R_{\mu}$ is a maximal ideal of $B$ note that $Q_{\mu}$ is the maximal ideal of $C_{\mu}$. Let $t$ be the canonical homomorphism of $C_{\mu}$ onto its residue class field, $C_{\mu} / Q_{\mu} \cdot t \mid B$ is a homomorphism of $B$ into $C_{\mu} / Q_{\mu}$ having as kernel $R_{\mu}$. Since $C_{\mu}=B_{Q_{\mu}}$ it is easy to see that $t \mid B$ maps $B$ onto $C_{\mu} / Q_{\mu}$; thus $R_{\mu}$ is maximal, proving the theorem. 
Theorem 5.21. Specm $B=\left\{R_{\mu}: \mu \in \delta_{B} X\right\}$.

Proof. Let $M^{\prime} \in \operatorname{specm} B$ and let $P \equiv M^{\prime} \cap A$; then $P \in \operatorname{spec} A$. Since $B \neq F$ there exists $f \in M^{\prime}-0$. Let $f=a / b$, where $a, b \in A$ and $b \neq 0$; then $b f=a \in M^{\prime}$. Since $f \neq 0, a \neq 0$, thus $a \in P-0$, and so $P \neq 0$. Henriksen has shown [12] that $P$ is local; thus there exists a unique $\mu \in \delta X$ so that $P \subset M_{\mu}$. Since $M^{\prime}$ is proper, $M^{\prime} \cap U(B)=\varnothing, P \cap U(B)=\varnothing$, and so $\nu_{\mu}(a)>\gamma_{\mu}$, for all $\gamma_{\mu} \in \Gamma_{\mu}$. Hence $-\nu_{\mu}(a)<h(\mu)$ and so we see that $h(\mu)>$ $-\infty_{\mu}$ and thus $\mu \in \delta_{B} X$. As a consequence, $M^{\prime} \subset R_{\mu}$. Since $M^{\prime}$ is maximal, $M^{\prime}=R_{\mu}$. Using (5.20) the rest of the theorem is proved.

The inclusion map 1 of $A$ into $B$ induces continuous map $1^{*}$ of $\operatorname{spec} B$ into $\operatorname{spec} A$. Although $\operatorname{spec} B$ is compact we cannot conclude that $1^{*}(\operatorname{spec} B)$ is closed in $\operatorname{spec} A, \operatorname{since} \operatorname{spec} A$ is not a Hausdorff space. Given $M^{\prime} \in \operatorname{specm} B$ we have constructed a map, in the proof of (5.21), of $M^{\prime}$ to $\mu \in \delta_{B} X$ such that $M^{\prime}=R_{\mu}$, and shown that the map $R: \mu \in \delta_{B} X \mapsto R_{\mu} \in \operatorname{specm} B$ is bijective. Examples abound to show that $\delta_{B} X$ need not be a closed set of $\delta X$. We will give a fairly general construction from which many such examples can be constructed. Let $V$ be any nonempty subset of $\delta X$. For each $\mu \in V$ let $P_{\mu}$ be a nonzero prime ideal of $A$ that is contained in $M_{\mu}$. Let $B \equiv$

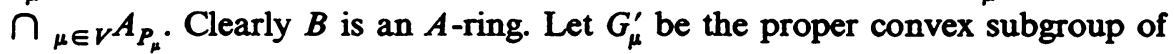
$G_{\mu}$ that corresponds to $P_{\mu}$ (using Krull's theorem [17]), for $\mu \in V$. Let $k(\mu) \equiv \inf G_{\mu}^{\prime}$, for each $\mu \in V$, and let $k(\mu)=-\infty_{\mu}$ for all $\mu \in \delta X-V$, $k \in H$, and $V=\left\{\mu \in \delta X: k(\mu)>-\infty_{\mu}\right\}$. Let $h \equiv$ $\wedge_{a \in J(k)} \tilde{a}$; then $h \in \mathrm{afa}(H)$, and $h \geqslant k$. Clearly $\nu(B)=J(k)$ and $J(k)=$ $J(h)$; thus $h=L \nu(B) . \delta_{B} X=\left\{\mu \in \delta X: h(\mu)>-\infty_{\mu}\right\}$, and hence $V \subset \delta_{B} X$.

EXAMPLe 5.3. Let $T$ be any subset of $X$ that is contained in a compact set $K$ of $X$, and let $V \equiv X-T$; then $B \equiv\{f \in F: f$ is regular on $V\}$ and $\delta_{B} X=(\delta X)-T . V \subset \delta_{B} X . \delta_{B} X$ is not closed in $\delta X$.

An analysis of the ideal theory of $B$, the theory of the sub $B$-modules of $F$, and the fractional ideals of $F$ with respect to $B$ can be made very much along the lines of that presented in $\$ \S 2-4$, using $\tau$ in place of $\nu$, as the reader can easily verify.

\section{REFERENCES}

1. N. L. Alling, The valuation theory of meromorphic function fields over open Riemann surfaces, Acta Math. 110 (1963), 79-96.

2. __ The valuation theory of meromorphic function fields, Proc. Sympos. Pure Math., vol. 11, Amer. Math. Soc., Providence, R. I., 1968, pp. 8-29.

3. 423-427.

4. B. Banaschewski, Zur Idealtheorie der ganzen Funktionen, Math. Nachr. 19 (1958), 136-160.

5. L. Bers, On rings of analytic functions, Bull. Amer. Math. Soc. 54 (1948), 311-315.

6. G. Birkhoff, Lattice theory, rev. ed., Amer. Math. Soc. Colloq. Publ., vol. 25, Amer. Math. Soc., Providence, R. I., 1948. 
7. H. Florack, Reguläre und meromorphe Funktionen ouf nicht geschlossenen Riemannschen Flächen, Schr. Math. Inst. Univ. Münster, no. 1 (1948).

8. L. Gillman and M. Jerison, Rings of continuous functions, Van Nostrand, Princeton, N. J., 1960.

9. R. C. Gunning and H. Rossi, Analytic functions of several complex variables, Prentice-Hall, Englewood Cliffs, N. J., 1965.

10. O. Helmer, Divisibility properties of integral functions, Duke Math. J. 6 (1940), 345-356.

11. M. Henriksen, On the ideal structure of the ring of entire functions, Pacific J. Math. 2 (1952), 179-184.

12. 711-720. , On the prime ideals of the ring of entire functions, Pacific J. Math. 3 (1953),

13. E. Hewitt, Rings of real-valued continuous functions. I, Trans. Amer. Math. Soc. 64 (1948), 45-99.

14. H. Iss'sa, On meromorphic function fields on a Stein variety, Ann. of Math. 83 (1966), 34-46.

15. J. Kelleher, Rings of meromorphic functions on noncompact Riemann surfaces, Canad. J. Math. 21 (1969), 284-300.

16. S. Kochen, Ultroproducts in the theory of models, Ann. of Math. 74 (1961), 221-261.

17. W. Krull, Allgemeine Bewertungstheorie, J. Reine Angew. Math. 167 (1931), 160-196.

18. O. F. G. Schilling, Ideal theory on open Riemann surfaces, Bull. Amer. Math. Soc. 52 (1946), 945-963.

19. O. Zariski and P. Samuel, Commutative algebra. I, Van Nostrand, Princeton, N. J., 1958.

20. Commutative algebra. II, Van Nostrand, Princeton, N. J., 1960.

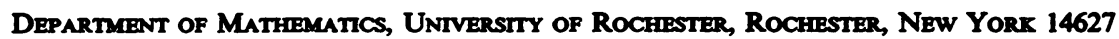

